\title{
Robust Calibration For SVI Model Arbitrage Free
}

\author{
Tahar FERHATI \\ tahar.ferhati@gmail.com \\ March 14, 2020 \\ (2nd Version)
}

\begin{abstract}
The purpose of this paper is to study the Stochastic Volatility Inspired model (SVI) as implied volatility model: we study the analytic part of the SVI with the arbitrage conditions, we establish the initial guess and the parameter's boundaries. Until recently it was not possible to find sufficient conditions that would guarantee an SVI model calibration arbitrage-free. The main contribution in this paper is that we provide two methods to resolve the arbitrage problem (butterfly and calendar spread): the first one is numerical using the Sequential Least-Squares Quadratic Programming (SLSQP) algorithm, and the second one is analytical by using sufficient conditions that guarantee an SVI arbitrage-free.

Our method guarantee to get SVI calibration with butterfly and calendar spread arbitrage-free, We provide many numerical examples with arbitrage such as Vogt Axel example and we show how to fix them. The calibration method is tested on 23 equity indexes with 14 maturities each and we get 322 slices fits using the same initial guess and the SVI parameters boundaries for all indexes.

This new calibration method is very important and it meets practical need: resolving this arbitrage problem will pave the way to the surface calibration and the transition from implied volatility to local volatility using Dupire's formula, therefore, it allows price different kind of pathdependent options such as barrier options, and American options. The SVI model could also be applied to price interest rate derivatives such as swaptions, interest rate caps, and floors.
\end{abstract}

Keywords: Implied/Local Volatility, SVI, Arbitrage-Free,butterfly spread, calendar spread, Calibration, Quadratic Programming. 


\section{Contents}

$\begin{array}{lr}\text { Introduction } & \mathbf{3}\end{array}$

1 Local and Implied Volatility $\quad \mathbf{5}$

1.1 Local Volatility . . . . . . . . . . . . . . . . . . . . . . . . 5

1.1.1 Fokker-Planck equation and Dupire's PDE .............. 5

1.1.2 Dupire's Formula with Implied Volatility . . . . . . . . . . . . . . . . . . 7

1.2 The Roger Lee's Moment Formula . . . . . . . . . . . . . . . . . . . . . . . . . . 8

1.2.1 The Large-Strike Tail . . . . . . . . . . . . . . . . . . . . . . 8

1.2 .2 The Small-Strike Tail . . . . . . . . . . . . . . . . . . . . . . 9

1.3 Convergence Heston Model to SVI . . . . . . . . . . . . . . . . . . . . . . . . 10

2 Stochastic Volatility Inspired SVI 11

2.1 History of SVI . . . . . . . . . . . . . . . . . . . . . . . 11

2.2 SVI Model Formulation . . . . . . . . . . . . . . . . . . . . . . . 11

2.2.1 The raw SVI parameterization . . . . . . . . . . . . . . . 11

2.2.2 The natural SVI parameterization . . . . . . . . . . . . . . 13

2.2.3 The SVI Jump-Wings (SVI-JW) Parameterization . . . . . . . . . . . . . . 14

2.3 Characterisation of Static Arbitrage . . . . . . . . . . . . . . . . . 14

2.3.1 RND tests for arbitrage . . . . . . . . . . . . . . . . . . . . . . 15

2.3.2 Calendar Spread Arbitrage . . . . . . . . . . . . . . . 15

2.3 .3 Butterfly arbitrage . . . . . . . . . . . . . . . . . . 16

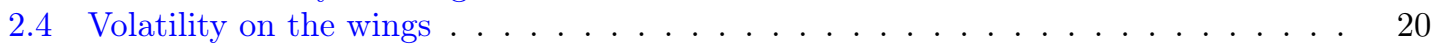

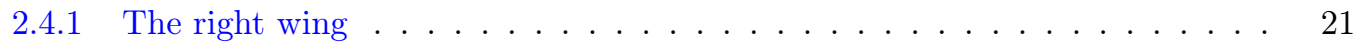

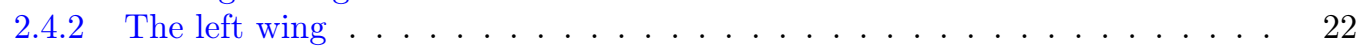

3 Robust Calibration For SVI Arbitrage Free $\quad 24$

3.1 The Raw SVI Calibration . . . . . . . . . . . . . . . . . . . . . . . . . 24

3.2 SVI's Parameters Boundaries . . . . . . . . . . . . . . . . . . . . . . 25

3.2 .1 The Initial Guess . . . . . . . . . . . . . . . . . . . . . . . 27

3.2 .2 The Butterfly Arbitrage Constraints . . . . . . . . . . . . . . . . . . 27

3.3 Input Data . . . . . . . . . . . . . . . . . . . . . . . . . . . . . 28

3.4 Sequential Quadratic Programming For SVI Calibration . . . . . . . . . . . . . . 29

3.4.1 The Nonlinear Programming Problem NLP . . . . . . . . . . . . . . . 30

3.5 Numerical Applications . . . . . . . . . . . . . . . . . . . . 34

3.5.1 Some Arbitrage Examples . . . . . . . . . . . . . . . . . . . . . . . 34

3.5.2 Equity Indexes Calibration . . . . . . . . . . . . . . . . . . . . . . . . . . . 35

3.5.3 Multi-Slices SVI Calibration . . . . . . . . . . . . . . . . 38

3.6 SVI Calibration with Weights . . . . . . . . . . . . . . . . 40

4 Conclusion $\quad 43$

$\begin{array}{ll}\text { A SVI Calibration Using Indexes } & 44\end{array}$

$\begin{array}{lr}\text { Bibliography } & 51\end{array}$ 


\section{Introduction}

The implied volatility is an important element used for pricing and hedging in the financial market. It can be obtained by inverting Black-Scholes formula (1973) for a given strike and maturity. These values are explicitly available only for some strikes and maturities. For this reason we need a model that allow to obtain an accurate values close to those observed in the market.

Many interpolation techniques has been proposed in the last decades, we can class them in two categories: mathematical interpolation models and financial interpolation models. The appropriate model should respect some arbitrage free condition.

Many authors in the past such as Dupire (1994) [1], Derman and Kani (1994) [2], and Rubinstein (1994) tried to model local volatility, we propose here an overview of the more recent methods used to resolve this problem.

Kahalé (2004) [3] presented an interpolation method for implied volatilities in the equity and forex markets using one-dimensional interpolation algorithm with smoothness properties. Kahalé assumes arbitrage-free in the input market volatilities and computes an interpolating surface for all strikes and maturities in three-step procedure: in the first step he interpolates the (call) price for each maturity using piecewise convex polynomials. Hence, the call price function obtained is arbitrage free and after he calculates the implied volatility by inverting the BS formula, in the second step he interpolates linearly the total implied variance. Finally, he makes some adjustments to the call prices to ensure that IVS is globally arbitrage-free.

Jim Gatheral (2004) presented for the first time the Stochastic Volatility Inspired model (SVI) in the Global Derivatives and Risk Management conference in Madrid.

Benko et al.(2007) [4] applied non-parametric smoothing methods to estimate the implied volatility (IV). They combine the IV smoothing with the state-price density (SPD) estimation in order to correct the arbitrage condition reflected by the non-positive SPD, for this, they used the local polynomial smoothing technique.

Fengler (2009) [5] proposed an approach for smoothing the implied volatility smile and provides a methodology for arbitrage free interpolation. His methodology consists in using the natural smoothing splines under suitable shape constraints. This method works even when the input data is not arbitrage free.

Andreasen-Huge (2010) [6] presented an interpolation and extrapolation method of European option prices based on a one step implicit finite difference Euler scheme applied to a local volatility parametrization.

Glaser and Heider (2012)[7] use locally constrained least squares approximations to construct the arbitrage-free call price surfaces. They calculate derivatives of the call surface to obtain implied volatility, local volatility and transition probability density.

Fingler-Hin (2013) [8] use semi-nonparametric estimator for the entire call price surface based on a tensor-product B-spline.

Gatheral \& Jacquier (2014) [9] presents the stochastic volatility inspired model (SVI) a parametric model of the implied volatility smile. The model fits very well the equity market, however, no condition is known that could guarantee the arbitrage free of the model. This is the main challenge in our project.

Several problems arise in the past regarding the calibration of the SVI and simultaneously avoiding arbitrage. Our objective is the analytic study of the SVI model, calibration of the model and avoiding the arbitrage.

The rest of this paper is organized as follows: in the first chapter we give a brief introduction of the local and implied volatility and we show the relation between them in the case of the SVI model. Finally, we show the large-time asymptotic convergence of the Heston implied volatility to SVI model. 
In chapter 2, we present the SVI model formulation and the different forms such as the natural SVI, Jump-Wings SVI. We also define the characterization of static arbitrage: calendar spread and butterfly. We provide for the first time an analytical sufficient conditions that guarantee an SVI model arbitrage-free.

In chapter 3, we present a new robust calibration method for the SVI model using Sequential Quadratic Programming (SQP) optimization method that eliminate automatically the arbitrage during during the calibration ( even in the case where our input data contain arbitrage). We explain the method, and we establish the SVI's parameter boundaries and the initial guess. We illustrate the performance of our algorithm in two numerical examples with arbitrage, one of them in the Axel Vogt example.

Finally, we apply our calibration method to calibrate the implied volatility coming from 23 indexes with 14 maturities each (322 slices). We use also calibration with weights to improve the performance of fitting. 


\section{Chapter 1}

\section{Local and Implied Volatility}

In this Chapter we provide brief introduction and summary of the relation between stochastic and the local volatility. This tools are the most important ingredient used in the pricing of exotic equity options. An excellent lecture note by Gatheral in [10] will be followed as reference for this section.

The local volatility allows to explain why for a different strikes and maturities we can get different prices in the Black-Scholes formula. As the distribution of the stock price returns is characterized by high peak and fat-tailed compared to the normal distribution, this motivate to model variance as a random variable which is a consequence of the mean reversion of volatility.

\subsection{Local Volatility}

The local volatility is used to price exotic options, Breeden and Litzenberger in [11] proved for the first time that the risk neutral density RND could be calculated using the market price of European options. Dupire (1994) [1], Derman and Kani (1994) mentioned that there exist a unique diffusion process consistent with these distribution. This unique state-dependent diffusion coefficient is called the local volatility function $\sigma_{L}(S, t)$.

Dupire, Derman and Kani represent local volatilities as an average over all instantaneous volatilities in a stochastic volatility world.

Dumas, Fleming, and Whaley (1998) confirmed by empirical analysis that the dynamic of implied volatility surface is not consistent with the assumption of constant local volatilities.

\subsubsection{Fokker-Planck equation and Dupire's PDE}

We consider an underlying asset that verify the following SDE

$$
d S_{t}=S_{t}(r-q) d t+S_{t} \sigma\left(S_{t}, t\right) d W_{t}
$$

The Fokker-Planck's equation is therefore

$$
\partial_{t} P\left(S_{t}, t\right)=-\partial_{S}\left((r-q) S_{t} P\left(S_{t}, t\right)\right)+\partial_{S S}\left(\frac{\sigma\left(S_{t}, t\right)^{2}}{2} S_{t}^{2} P\left(S_{t}, t\right)\right)
$$

Where $p(., t)$ is the density of the underlying asset price at time $t$. Let's note $C(K, T)$ the price of option with strike $k$, maturity $T$ at $t_{0}$.

$$
C(K, T)=e^{-r\left(T-t_{0}\right)} \int_{0}^{\infty}\left(S_{T}-K\right)_{+} P\left(S_{T}, T\right) d S
$$

We derive both sides of the equation with respect to $t$ and we use (1.2), we obtain

$$
\begin{aligned}
\partial_{t} C(K, T)= & -r C(K, T)+e^{-r\left(T-t_{0}\right)} \int_{0}^{\infty}\left(S_{T}-K\right)_{+}\left[-\partial_{S}((r-q) S P(S, T))\right. \\
& \left.+\partial_{S S}\left(\frac{1}{2} \sigma(S, T)^{2} S^{2} P(S, T)\right)\right] d S
\end{aligned}
$$


We use the integration by parts

$$
\begin{aligned}
\partial_{t} C(K, T)= & -r C(K, T)-e^{-r\left(T-t_{0}\right)} \int_{K}^{\infty} \partial_{S}\left(\frac{1}{2} \sigma^{2}(S, T) S^{2} P(S, T)\right) d S \\
& +e^{-r\left(T-t_{0}\right)} \int_{0}^{\infty}(r-q)\left(S_{T}-K\right)_{+} P(S, T) d S \\
& +K(r-q) e^{-r\left(T-t_{0}\right)} \int_{K}^{\infty} P(S, T) d S \\
\partial_{t} C(K, T)= & -r C(K, T)+e^{-r\left(T-t_{0}\right)} \frac{1}{2} \sigma^{2}(K, T) K^{2} P(K, T) \\
& +(r-q) e^{-r\left(T-t_{0}\right)} \int_{0}^{\infty}\left(S_{T}-K\right)_{+} P(S, T) d S \\
& +K(r-q) e^{-r\left(T-t_{0}\right)} \int_{K}^{\infty} P(S, T) d S
\end{aligned}
$$

We have also

$$
\begin{gathered}
C(K, T)=e^{-r\left(T-t_{0}\right)} \int_{0}^{\infty}\left(S_{T}-K\right)_{+} p(S, T) d S \\
\partial_{K} C(K, T)=-e^{-r\left(T-t_{0}\right)} \int_{K}^{\infty} p(S, T) d S \\
\partial_{K K} C(K, T)=e^{-r\left(T-t_{0}\right)} p(K, T)
\end{gathered}
$$

We replace in (1.6) and we obtain

$$
\begin{aligned}
\partial_{t} C(K, T)= & -r C(K, T)+\frac{1}{2} \sigma^{2}(K, T) K^{2} \partial_{K K} C(K, T) \\
& +(r-q) C(K, T)-(r-q) K \partial_{K} C(K, T)
\end{aligned}
$$

Finally we get the Dupire's PDE

$$
\frac{\partial C}{\partial T}(K, T)=-q C(K, T)-(r-q) K \frac{\partial C}{\partial K}(K, T)+\frac{1}{2} \sigma^{2}(K, T) K^{2} \frac{\partial^{2} C}{\partial K^{2}}(K, T)
$$

With the boundary conditions

$$
\begin{cases}C\left(K, t_{0}\right)=\max \left(S_{0}-K, 0\right) & \text { for } 0 \leq K \\ \lim _{K \rightarrow 0} C(K, T)=S_{0} e^{-q\left(T-t_{0}\right)} & \text { for } t_{0} \leq T \\ \lim _{K \rightarrow+\infty} C(K, T)=0 & \text { for } t_{0} \leq T\end{cases}
$$

Where $r(t)$ is the risk-free rate, and $q$ is the dividend yield.

We can express the option price as a function of the forward price

$$
F_{T}=S_{0} \exp \left\{\int_{0}^{T} \mu(t) d t\right\}
$$

where $\mu(t)=r-q$ is the risk-neutral drift of the stock price process.

Finally, we would get the same expression minus the drift term as following

$$
\frac{\partial C}{\partial T}(K, T)=\frac{1}{2} \sigma^{2}(K, T) K^{2} \frac{\partial^{2} C}{\partial K^{2}}(K, T)
$$

Therefore;

$$
\sigma_{l o c}^{2}\left(K, T, S_{0}\right)=\frac{\frac{\partial C}{\partial T}(K, T)}{\frac{1}{2} K^{2} \frac{\partial^{2} C}{\partial K^{2}}(K, T)}
$$




\subsubsection{Dupire's Formula with Implied Volatility}

Gatheral explained in his book [12] how we find the relation that combine local volatility and implied volatility, it allows the transformation from one to the other. Therefore, we can write the call price formula in terms of the implied total variance $w$.

Let's consider the following call price

$$
C\left(S_{0}, K, T\right)=C_{B S}\left(S_{0}, K, \sigma_{B S}\left(S_{0}, K, T\right), T\right)
$$

Where $\sigma_{B S}\left(K, T ; S_{0}\right)$ is the Black-Scholes implied volatility. The implied total variance is defined by:

$$
\mathrm{w}\left(S_{0}, K, T\right) \equiv \sigma_{B S}^{2}\left(S_{0}, K, T\right) T
$$

and the logforward moneyness is:

$$
x=\ln \left(\frac{K}{F_{T}}\right), \text { with } \quad F_{T}=S_{0} \exp \left\{\int_{0}^{T} d t \mu(t)\right\}
$$

The Black-Scholes formula with this variable change becomes

$$
\begin{aligned}
C_{B S}\left(F_{T}, x, \mathrm{w}\right) & =F_{T}\left\{N\left(d_{1}\right)-e^{x} N\left(d_{2}\right)\right\} \\
& =F_{T}\left\{N\left(-\frac{x}{\sqrt{\mathrm{w}}}+\frac{\sqrt{\mathrm{W}}}{2}\right)-e^{x} N\left(-\frac{x}{\sqrt{\mathrm{w}}}-\frac{\sqrt{\mathrm{w}}}{2}\right)\right\}
\end{aligned}
$$

The Dupire equation in (1.11) will be

$$
\frac{\partial C}{\partial T}=\frac{v_{L}}{2}\left\{\frac{\partial^{2} C}{\partial x^{2}}-\frac{\partial C}{\partial x}\right\}+\mu(T) C
$$

Where the local variance is: $v_{L}=\sigma^{2}\left(S_{0}, K, T\right)$.

Now we calculate the derivatives of the Black-Scoles formula in (1.17)

$$
\begin{gathered}
\frac{\partial^{2} C_{B S}}{\partial \mathrm{W}^{2}}=\left(-\frac{1}{8}-\frac{1}{2 \mathrm{w}}+\frac{x^{2}}{2 \mathrm{w}^{2}}\right) \frac{\partial C_{B S}}{\partial \mathrm{w}} \\
\frac{\partial^{2} C_{B S}}{\partial x \partial \mathrm{w}}=\left(\frac{1}{2}-\frac{x}{\mathrm{w}}\right) \frac{\partial C_{B S}}{\partial \mathrm{w}} \\
\frac{\partial^{2} C_{B S}}{\partial x^{2}}-\frac{\partial C_{B S}}{\partial x}=2 \frac{\partial C_{B S}}{\partial \mathrm{w}}
\end{gathered}
$$

We rewrite (1.18) in terms of the implied variance $w$ and we get

$$
\begin{gathered}
\frac{\partial C}{\partial x}=\frac{\partial C_{B S}}{\partial x}+\frac{\partial C_{B S}}{\partial \mathrm{w}} \frac{\partial \mathrm{w}}{\partial x} \\
\frac{\partial^{2} C}{\partial x^{2}}=\frac{\partial^{2} C_{B S}}{\partial x^{2}}+2 \frac{\partial^{2} C_{B S}}{\partial x \partial w} \frac{\partial \mathrm{W}}{\partial x}+\frac{\partial^{2} C_{B S}}{\partial \mathrm{W}^{2}}\left(\frac{\partial \mathrm{w}}{\partial x}\right)^{2}+\frac{\partial C_{B S}}{\partial \mathrm{w}} \frac{\partial^{2} \mathrm{~W}}{\partial x^{2}} \\
\frac{\partial C}{\partial T}=\frac{\partial C_{B S}}{\partial T}+\frac{\partial C_{B S}}{\partial \mathrm{w}} \frac{\partial \mathrm{w}}{\partial T}=\frac{\partial C_{B S}}{\partial \mathrm{w}} \frac{\partial \mathrm{w}}{\partial T}+\mu(T) C_{B S}
\end{gathered}
$$

Note that the dependence on time $T$ in (1.17) is only in the term of the forward price $F_{T}$. Equation (1.11) becomes

$$
\begin{gathered}
\frac{\partial C_{B S}}{\partial \mathrm{w}} \frac{\partial \mathrm{w}}{\partial T} \\
=\frac{v_{L}}{2}\left\{-\frac{\partial C_{B S}}{\partial x}+\frac{\partial^{2} C_{B S}}{\partial x^{2}}-\frac{\partial C_{B S}}{\partial w} \frac{\partial \mathrm{w}}{\partial x}+2 \frac{\partial^{2} C_{B S}}{\partial x \partial \mathrm{w}} \frac{\partial \mathrm{w}}{\partial x}+\frac{\partial^{2} C_{B S}}{\partial \mathrm{w}^{2}}\left(\frac{\partial \mathrm{w}}{\partial x}\right)^{2}+\frac{\partial C_{B S}}{\partial \mathrm{w}} \frac{\partial^{2} \mathrm{w}}{\partial x^{2}}\right\} \\
=\frac{v_{L}}{2} \frac{\partial C_{B S}}{\partial \mathrm{w}}\left\{2-\frac{\partial \mathrm{w}}{\partial x}+2\left(\frac{1}{2}-\frac{x}{\mathrm{w}}\right) \frac{\partial \mathrm{w}}{\partial x}+\left(-\frac{1}{8}-\frac{1}{2 \mathrm{w}}+\frac{y^{2}}{2 \mathrm{w}^{2}}\right)\left(\frac{\partial \mathrm{w}}{\partial x}\right)^{2}+\frac{\partial^{2} \mathrm{w}}{\partial x^{2}}\right\}
\end{gathered}
$$


After simplification we get

$$
\frac{\partial \mathrm{W}}{\partial T}=v_{L}\left\{1-\frac{x}{\mathrm{w}} \frac{\partial \mathrm{w}}{\partial x}+\frac{1}{4}\left(-\frac{1}{4}-\frac{1}{\mathrm{w}}+\frac{x^{2}}{\mathrm{w}^{2}}\right)\left(\frac{\partial \mathrm{w}}{\partial x}\right)^{2}+\frac{1}{2} \frac{\partial^{2} \mathrm{w}}{\partial x^{2}}\right\}
$$

Finally, the local volatility expression in terms of the total variance ( the implied volatility) is

$$
v_{L}=\frac{\frac{\partial \mathrm{w}}{\partial T}}{1-\frac{x}{\mathrm{w}} \frac{\partial \mathrm{w}}{\partial x}+\frac{1}{4}\left(-\frac{1}{4}-\frac{1}{\mathrm{w}}+\frac{x^{2}}{\mathrm{w}^{2}}\right)\left(\frac{\partial \mathrm{w}}{\partial x}\right)^{2}+\frac{1}{2} \frac{\partial^{2} \mathrm{w}}{\partial x^{2}}}=\frac{\frac{\partial \mathrm{w}}{\partial T}}{g(x)}
$$

With;

$$
\mathrm{w}\left(S_{0}, K, T\right) \equiv \sigma_{B S}^{2}\left(S_{0}, K, T\right) T
$$

and;

$$
g(x)=1-\frac{x}{\mathrm{w}} \frac{\partial \mathrm{w}}{\partial x}+\frac{1}{4}\left(-\frac{1}{4}-\frac{1}{\mathrm{w}}+\frac{x^{2}}{\mathrm{w}^{2}}\right)\left(\frac{\partial \mathrm{w}}{\partial x}\right)^{2}+\frac{1}{2} \frac{\partial^{2} \mathrm{w}}{\partial x^{2}}
$$

We will see after, in the SVI calibration section, the relation between the $g(x)$ and the butterfly arbitrage condition. As a first condition, in order to get a positive local volatility, both numerator $\frac{\partial \mathrm{w}}{\partial T}$ and denominator $g(x)$ should to be strictly positive.

\subsection{The Roger Lee's Moment Formula}

Roger Lee shows in [13] that the total implied variance is bounded by a linear function in the extreme strikes, and the maximum slope of the total variance $\mathrm{w}\left(S_{0}, K, T\right) \equiv \sigma_{B S}^{2}\left(S_{0}, K, T\right) T$ is 2 . This propriety is very important and any interpolation model should be consistent with this condition in the large-strikes.

Roger shows the relation between the maximal finite moments of the underlying process and the gradient of the wings.

Let's define $F_{0}=E\left(S_{T}\right)$ the forward price of the payoff $S_{T}$, and the log forward moneyness x is

$$
x \equiv \log \left(K / F_{0}\right)
$$

$I(x)$ is the Black-Scholes implied volatility at moneyness $x$ that solve

$$
C(K(x))=C^{B S}(x, I(x))
$$

With;

$$
C^{B S}(x, \sigma):=B_{0}\left(F_{0} \Phi\left(d_{+}\right)-K(x) \Phi\left(d_{-}\right)\right), \quad d_{ \pm}:=\frac{-x}{\sigma \sqrt{T}} \pm \frac{\sigma \sqrt{T}}{2}
$$

\subsubsection{The Large-Strike Tail}

The Large-Strike tail is a reference to the right wing in the zone with large positive strike $K$, or $\log$ forward moneyness $x$ positive or OTM call.

First, we present an asymptotic formula for the implied volatility $I(x)$ with a large strike and next the moment formula.

Lemma: There exists $x^{*}>0$ such that for all $x>x^{*}$,

$$
I(x)<\sqrt{2|x| / T}
$$

Proof. We know that $C^{B S}(x, I(x))$ is strictly monotone function with respect to the second argument $I(x)$, hence, we need only to show the inequality

$$
C^{B S}(x, I(x))<C^{B S}(x, \sqrt{2|x| / T}) \quad x>x^{*}
$$

By dominated convergence, and as $E\left(S_{T}\right)<\infty$ the limit in the left side is

$$
\lim _{x \rightarrow \infty} C(K(x))=\lim _{K \rightarrow \infty} B_{0} E\left(S_{T}-K\right)^{+}=0
$$


Using L'Hopital's rule, and we get in the right side

$$
\lim _{x \rightarrow \infty} C^{B S}(x, \sqrt{2|x| / T})=B_{0} F_{0}\left[\Phi(0)-\lim _{x \rightarrow \infty} e^{x} \Phi(-\sqrt{2|x|})\right]=B_{0} F_{0} / 2
$$

Comparing (1.28) and (1.29), it's clearly that the relation in (1.27), and we conclude that:

$$
I(x)<\sqrt{2|x| / T}
$$

The interpretation of this lemma is: given the total implied variance

$$
\mathrm{w}\left(S_{0}, K, T\right) \equiv \sigma_{B S}^{2}\left(S_{0}, K, T\right) T=I(x)^{2} T<2|x|
$$

In the large strikes zone, the total variance in the right wing is linear and the maximum value for the slope is 2 .

\section{Theorem (The Moment Formula For the right wing)}

Let

$$
\tilde{p}:=\sup \left\{p: E S_{T}^{1+p}<\infty\right\} \quad \beta_{R}:=\limsup _{x \rightarrow \infty} \frac{I^{2}(x)}{|x| / T}
$$

Then;

$$
\beta_{R} \in[0,2] \quad \text { and } \quad \tilde{p}:=\sup \left\{p: E S_{T}^{1+p}<\infty\right\}=\frac{1}{2 \beta_{R}}+\frac{\beta_{R}}{8}-\frac{1}{2}
$$

and by inverting this, we get;

$$
\beta_{R}=2-4\left(\sqrt{\tilde{p}^{2}+\tilde{p}}-\tilde{p}\right)
$$

\subsubsection{The Small-Strike Tail}

As for the Large-Strike, we consider the Small-Strike for the left wing or small $K$ or negative $x$ or OTM put. We show that the tail slope is no larger than 2 .

Lemma : For any $\beta>2$ there exists $x^{*}$ such that for all $x<x^{*}$,

$$
I(x)<\sqrt{\beta|x| / T}
$$

For the limit case with $\beta=2$, we get the same inequality if $S_{T}$ satisfies $P\left(S_{T}=0\right)<1 / 2$

Proof. For $\beta>2$, there exists $x^{*}$ such that for all $x<x^{*}$,

$$
P\left(S_{T}<F_{0} e^{x}\right)<\Phi\left(-\sqrt{f_{-}(\beta)|x|}\right)-e^{-x} \Phi\left(-\sqrt{f_{+}(\beta)|x|}\right)
$$

The left hand is approximated to $P\left(S_{T}=0\right)$ for $x \rightarrow-\infty$, and the right side is approximated to 1 for $\beta>2$ and $1 / 2$ for $\beta=2$.

So we have for all $x<x^{*}$

$$
P^{B S}(x, I(x))=B_{0} E\left(K(x)-S_{T}\right)^{+} B_{0} K(x) P\left(S_{T}<F_{0} e^{x}\right)<P^{B S}(x, \sqrt{\beta|x| / T})
$$

Or, $P^{B S}(x, I(x))$ is strict monotone with respect to the second argument and we can conclude that;

$$
I(x)<\sqrt{\beta|x| / T}
$$

For $\beta=2$, and for the same reason of monotonicity of $P^{B S}(x, I(x))$, we get for all $x>x^{*}$

$$
B_{0} K(x) / 2>P^{B S}(x, \sqrt{2|x| / T})>B_{0} E\left(K(x)-S_{T}\right)^{+} B_{0} K(x) P\left(S_{T}=0\right)
$$

We obtain the result by dividing by $B_{0} K(x)$. 


\section{Theorem (The Moment Formula For the left wing)}

If we have;

$$
\tilde{q}:=\sup \left\{q: E S_{T}^{-q}<\infty\right\} \quad \beta_{L}:=\limsup _{x \rightarrow-\infty} \frac{I^{2}(x)}{|x| / T}
$$

Then; $\quad \beta_{L} \in[0,2] \quad$ and $\quad \tilde{q}=\frac{1}{2 \beta_{L}}+\frac{\beta_{L}}{8}-\frac{1}{2}$

By inverting $\tilde{q}$ we get

$$
\beta_{L}=2-4\left(\sqrt{\tilde{q}^{2}+\tilde{q}}-\tilde{q}\right)
$$

Where for $\tilde{q}=\infty, \beta_{L}=0$

\subsection{Convergence Heston Model to SVI}

Gatheral and Jacquier show in [14] the large-time asymptotic convergence of the Heston implied volatility to a sampler expression consistent with the Stochastic Volatility Inspired (SVI parameterization). In order to interpret the SVI parameters in terms of the implied volatility in the Heston model. SVI could be consider the limit of Heston model in the large maturity $(T \rightarrow \infty)$.

Let's consider the expression of the SVI parametrization

$$
\sigma_{S V I}^{2}(x)=\frac{\omega_{1}}{2}\left(1+\omega_{2} \rho x+\sqrt{\left(\omega_{2} x+\rho\right)^{2}+1-\rho^{2}}\right), \quad \text { for all } x \in R
$$

Where $\mathrm{x}$ is the log-froward moneyness.

We consider the Heston model where $\left(S_{t}\right)_{t \geq 0}$ follow the process

$$
\begin{aligned}
\mathrm{d} S_{t} & =\sqrt{v_{t}} S_{t} \mathrm{~d} W_{t}, S_{0} \in R_{+}^{*} \\
\mathrm{~d} v_{t} & =\kappa\left(\theta-v_{t}\right) \mathrm{d} t+\sigma \sqrt{v_{t}} \mathrm{~d} Z_{t}, v_{0} \in R_{+}^{*} \\
\mathrm{~d}\langle W, Z\rangle_{t} & =\rho \mathrm{d} t
\end{aligned}
$$

with $\rho \in[-1,1], \kappa, \theta, \sigma$ and $v_{0}$ are strictly positive and $2 \kappa \theta \geq \sigma^{2}$ (the Feller condition).

Proposition Under $\kappa-\rho \sigma>0$ assumption $\sigma_{S V I}^{2}(x)=\sigma_{\infty}^{2}(x)$ for all $x \in R$

Now we will interpret the SVI parameters in function of the Heston parameters model We proceed to the following parametrization

$$
\omega_{1}:=\frac{4 \kappa \theta}{\sigma^{2}\left(1-\rho^{2}\right)}\left(\sqrt{(2 \kappa-\rho \sigma)^{2}+\sigma^{2}\left(1-\rho^{2}\right)}-(2 \kappa-\rho \sigma)\right), \quad \text { and } \quad \omega_{2}:=\frac{\sigma}{\kappa \theta}
$$

and we find;

$$
\begin{aligned}
a & =\frac{\omega_{1}}{2}\left(1-\rho^{2}\right) \\
b & =\frac{\omega_{1} \omega_{2}}{2 T} \\
\tilde{\rho} & =\rho \\
m & =-\frac{\rho T}{\omega_{2}} \\
\tilde{\sigma} & =\frac{\sqrt{1-\rho^{2}} T}{\omega_{2}}
\end{aligned}
$$

Proof The proof of this proposition is voluntarily omitted and it could be found in [14] 


\section{Chapter 2}

\section{Stochastic Volatility Inspired SVI}

In this section, we will focus on the stochastic volatility inspired model SVI. We start by presenting the general framework of the model as presented by Jim Gatheral and Antoine Jacquier in [9]. Next, we describe the characterisation of static arbitrage which includes calender spread arbitrage and butterfly arbitrage. Finally, we calibrate the SVI model using real market data of different indexes such as the Eurostoxx 50, FTSIE, DAX...etc.

\section{$2.1 \quad$ History of SVI}

The stochastic volatility inspired model (SVI) was used for the first time at Merrill Lynch in 1999 by Jim Gatheral, and presented in 2004 at the annual conference of Global Derivatives in Madrid. As traders and practitioners require intuitive interpretation of the SVI parameters, Gatheral and Jacquier show in [14] that the SVI model could be the convergence of the total implied variance under Heston model for large enough maturity $T$, additionally, they provide an intuitive interpretation of SVI parameters.

The success of this model is due to its particular features:

1. For a fixed time to expiry $T$, the implied Black-Scholes variance $\sigma_{B S}^{2}(k, T)$ is linear, with respect to the $\log$ forward moneyness strike $k:=\log \left(K / F_{T}\right)$ as $|k| \rightarrow \infty$, where $K$ is the strike and $F_{T}$ is the forward price of the stock. This linearity is consistent with Roger Lee's moment formula [13].

2. It fits very well listed option prices, and careful choice of parameters allows for an arbitrage free interpolation.

\subsection{SVI Model Formulation}

The original SVI formulation is the so called the raw SVI presented in 2004. This formulation is very tractable. However, it's very difficult to find the precise condition to prevent arbitrage. We will present other formulation such as: the natural SVI parameterization and the SVI Jump-Wings (SVI-JW) parameterization.

\subsubsection{The raw SVI parameterization}

The raw SVI parameterization is a parametric model with 5 parameters $\chi_{R}=\{a, b, \rho, m, \sigma\}$, it's a model of the total implied variance $w\left(k ; \chi_{R}\right):=\sigma_{i m p}^{2}\left(k ; \chi_{R}\right) T$.

$$
w\left(k ; \chi_{R}\right)=a+b\left\{\rho(k-m)+\sqrt{(k-m)^{2}+\sigma^{2}}\right\}
$$

Where $k:=\log \left(\frac{K}{F_{T}}\right)$ is the $\log$ forward moneyness and $K$ is the strike.

For every $k \in \mathbb{R}$, and the set of the models parameters $\chi_{R}$ is given by 


$$
\begin{aligned}
a & \in \mathbb{R} \\
b & \geq 0 \\
|\rho| & <1 \\
m & \in \mathbb{R} \\
\sigma & >0 \\
a+b \sigma \sqrt{1-\rho^{2}} & \geq 0
\end{aligned}
$$

The last condition is calculated using the fact that the minimum of the total implied variance is positive; $w\left(k ; \chi_{R}\right) \geq 0$.

Note that some papers use the forward $\log$-moneyness or $\log$-strike $k:=\log \left(\frac{K}{F_{T}}\right)$ notation instead of using the strike $K$.

This choice is motivated by scaling reason: dividing the strike by the forward price and taking the logarithm does not change our computation. We adapt in the following section the notation of forward log-moneyness strike $k$.

$$
w\left(k ; \chi_{R}\right)=\sigma_{S V I}^{2}\left(k ; \chi_{R}\right)=a+b\left\{\rho(k-m)+\sqrt{(k-m)^{2}+\sigma^{2}}\right\}
$$

In practical applications, the model parameters are adopted to each option expiry. For a fixed maturity $T$, the smile (or slice) is a function $\sigma(k, t)$, this function is modeled by

$$
w\left(k ; \chi_{R}\right):=\sigma_{\text {imp }}^{2}\left(k ; \chi_{R}\right) T .
$$

We define also:

- The total implied variance: $w(k, T)=T \sigma_{B S}^{2}(k, T)$

- The implied variance: $v(k, T)=\sigma_{\mathrm{BS}}^{2}(k, T)=w(k, T) / T$

- The map of the volatility surface: $(k, T) \longmapsto w(k, T)$

- The slice function: for any fixed expiry $T>0, k \mapsto w(k, T)$

Now we consider the effects and the interpretation of changing the parameters $\chi_{R}=\{a, b, \rho, m, \sigma\}$ as describes in the figure (2.1).

- $a$ : determines the overall level of variance: an increasing $a$ increases the general level of variance, a vertical translation of the smile.

- $b$ : controls the angle between the left and right asymptotes: Increasing $b$ increases the slopes of both the put and call wings, tightening the smile.

- $\rho$ : determines the orientation of the graph: increasing $\rho$ decreases the slope of the left wing, and increases the slope of the right wing a counter-clockwise rotation of the smile.

- $m$ : translates the graph: increasing $m$ translates the smile to the right.

- $\sigma$ : determines curvature of the smile: Increasing $\sigma$ reduces the at-the-money (ATM) curvature of the smile.

The total implied variance $w\left(k ; \chi_{N}\right)$ has the left and right asymptotes that respect the assumption of linear wings, this result is consistent with the Roger Lee's moment formula mentioned above:

$$
\begin{array}{ll}
W_{L}(k)=a+b(\rho-1)(k-m) & k \rightarrow-\infty \\
W_{R}(k)=a+b(\rho+1)(k-m) & k \rightarrow \infty
\end{array}
$$



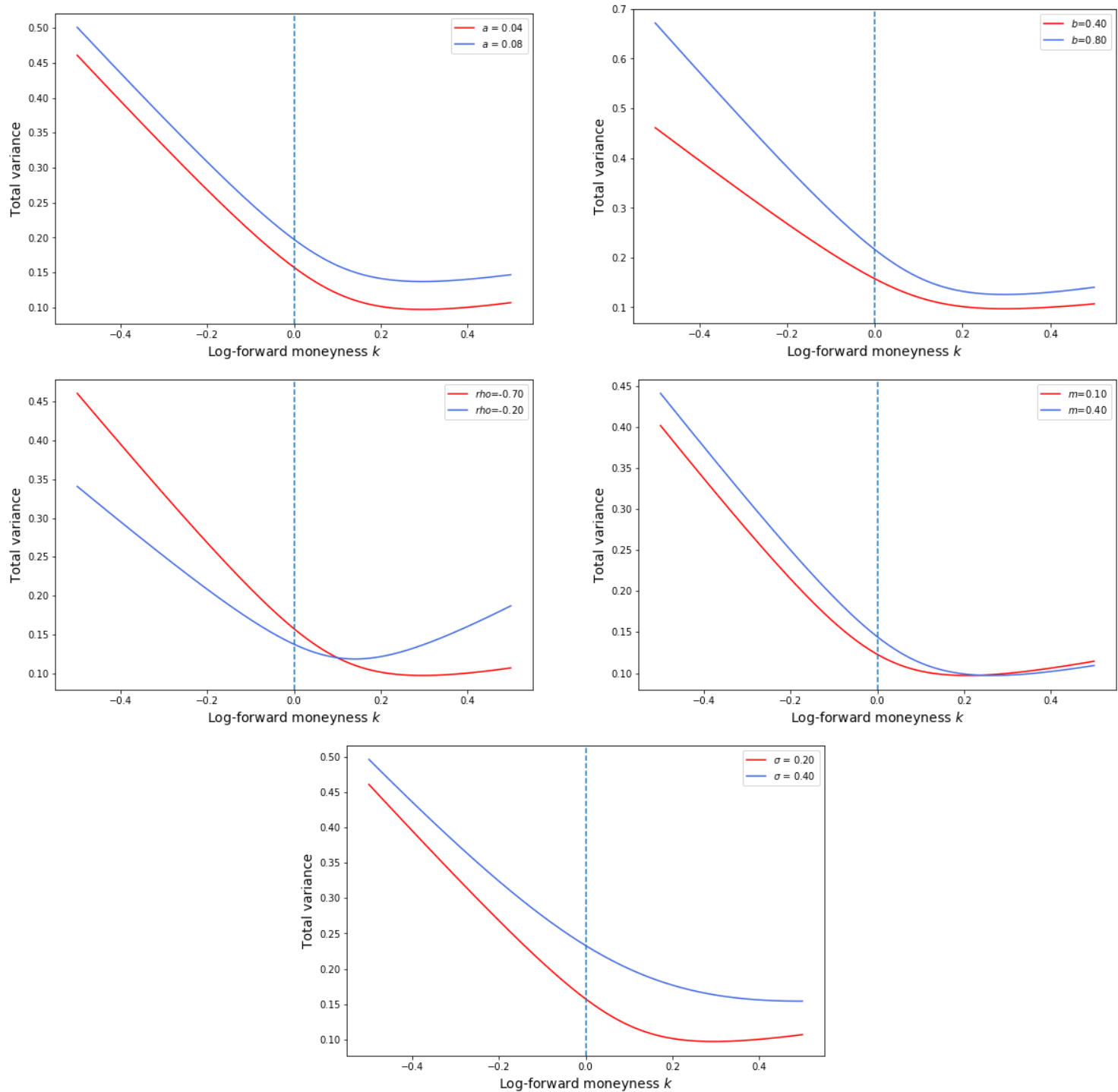

Figure 2.1: The effects of the parameters $\chi_{R}=\{a, b, \rho, m, \sigma\}$ in SVI model

\subsubsection{The natural SVI parameterization}

Gatheral and Jacquier propose in [9] another formulation of the natural SVI parameterization, it describes the total implied variance with parameters $\chi_{N}=\{\Delta, \mu, \rho, \omega, \zeta\}$.

$$
w\left(k ; \chi_{N}\right)=\Delta+\frac{\omega}{2}\left\{1+\zeta \rho(k-\mu)+\sqrt{(\zeta(k-\mu)+\rho)^{2}+\left(1-\rho^{2}\right)}\right\}
$$

with $\omega \geq 0, \Delta \in \mathbb{R}, \mu \in \mathbb{R},|\rho|<1$ and $\zeta>0$

We can show the relationship between the raw and the natural SVI model.

Lemma The parametrisation between the raw and the natural SVI is given by

$$
(a, b, \rho, m, \sigma)=\left(\Delta+\frac{\omega}{2}\left(1-\rho^{2}\right), \frac{\omega \zeta}{2}, \rho, \mu-\frac{\rho}{\zeta}, \frac{\sqrt{1-\rho^{2}}}{\zeta}\right)
$$

and the inverse mapping between the natural and the raw SVI is

$$
(\Delta, \mu, \rho, \omega, \zeta)=\left(a-\frac{\omega}{2}\left(1-\rho^{2}\right), m+\frac{\rho \sigma}{\sqrt{1-\rho^{2}}}, \rho, \frac{2 b \sigma}{\sqrt{1-\rho^{2}}}, \frac{\sqrt{1-\rho^{2}}}{\sigma}\right)
$$




\subsubsection{The SVI Jump-Wings (SVI-JW) Parameterization}

The SVI-Jump-Wings (SVI-JW) is parameterization of the implied variance $v(k, T)$ rather than the implied total variance $w(k, T)$. This kind of parameterization is intuitive for traders, and the parameters have a financial meaning. The model's parameterization was inspired by a similar parameterization attributed by Gatheral to Tim Klassen.

For a fixed time to expiry $t>0$, and a parameters set $\chi_{J}=\left\{v_{t}, \psi_{t}, p_{t}, c_{t}, \widetilde{v}_{t}\right\}$, the (SVI-JW) parameters defined from the row SVI is given by

$$
\begin{aligned}
v_{t} & =\frac{a+b\left\{-\rho m+\sqrt{m^{2}+\sigma^{2}}\right\}}{t} \\
\psi_{t} & =\frac{1}{\sqrt{w_{t}}} \frac{b}{2}\left(-\frac{m}{\sqrt{m^{2}+\sigma^{2}}}+\rho\right) \\
p_{t} & =\frac{1}{\sqrt{w_{t}}} b(1-\rho) \\
c_{t} & =\frac{1}{\sqrt{w_{t}}} b(1+\rho) \\
\tilde{v}_{t} & =\frac{1}{t}\left(a+b \sigma \sqrt{1-\rho^{2}}\right)
\end{aligned}
$$

Setting $w_{t}:=v_{t} t$, we notice that this parametrization dependency on time to expiration $\mathrm{t}$, hence the (SVI-JW) could be view as a generalisation of the raw SVI.

The SVI-JW parameters interpretation is as following :

- $v_{t}$ is the ATM variance.

- $p_{t}$ is the slope of the left (put) wing.

- $c_{t}$ is the slope of the right (call) wing.

- $v_{t}$ is the minimum implied variance.

\subsection{Characterisation of Static Arbitrage}

In this section we present the definition of static arbitrage in volatility and the necessary conditions to prevent it in our model. A static arbitrage is an arbitrage that does not require rebalancing of positions.

The total implied variance $w(\cdot, t)$ should satisfy the following conditions to be arbitrage free as presented in the following theorem [15].

Theorem 3.3 If the two-dimensional map $w: \mathbb{R} \times \mathbb{R}_{+} \rightarrow \mathbb{R}_{+}$satisfies:

(i) $w(\cdot, t)$ is of class $\mathcal{C}^{2}(\mathbb{R})$ for each $t \geq 0$

(ii) $w(k, t)>0$ for all $(k, t) \in \mathbb{R} \times \mathbb{R}_{+}^{*}$

(iii) $w(k, \cdot)$ is non-decreasing for each $k \in \mathbb{R}$

(iv) for each $(k, t) \in \mathbb{R} \times \mathbb{R}_{+}^{*}$, probability density function $P(k)$, is non-negative

(v) $w(k, 0)=0$ for all $k \in \mathbb{R}$

(vi) $\lim _{k \uparrow \infty} d_{+}(k, w(k, t))=-\infty$, for each $t>0$

Then the corresponding call price surface $(K, t) \mapsto \mathrm{BS}(K, w(\log (K), t))$ is free of static arbitrage.

Definition 3.3. Let $w: \mathbb{R} \times \mathbb{R}_{+}^{*} \rightarrow \mathbb{R}_{+}$be a two-dimensional map satisfying Theorem $3.3(\mathrm{i})-(\mathrm{ii})$

- $w$ is said to be free of calendar spread arbitrage if condition (iii) in Theorem 3.3 holds;

- $w$ is said to be free of butterfly arbitrage if condition (iv) in Theorem 3.3 holds. 
To check the arbitrage as explained in [16] and [17], we have to verify that the risk neutral density (RND) produces a volatility curve that is arbitrage-free. This RND will be used to calculate the price of our call/put options that should be also arbitrage-free.

There are two ways to check for arbitrage:

1. The risk neutral density (RND) test for butterfly arbitrage.

2. The test based on option strategies for butterfly and calendar spread arbitrage.

Breeden and Litzenberger [11], derive an expression of the discounted risk neutral density $f_{S_{T}}\left(S^{*}\right)$ as function of the second derivative of the call price $C(K)$ with respect to the strike $K$

$$
e^{-r T} f_{S_{T}}\left(S^{*}\right)=\left.\frac{\partial^{2} C}{\partial K^{2}}\right|_{K=S^{*}}
$$

\subsubsection{RND tests for arbitrage}

To satisfy this type of test we have to check the most common feature for a density function:

- Using the RND, we can obtain the call/put prices observed in the market by numerical integration of the RND.

- The RND is a positive function and its integral is equal to the one.

- Using the call price function $C(K)$, with strike $K$, the RND should produce monotonically decreasing call option prices with respect to the strike $K$.

The call price $C(K)$ is

$$
C(K)=e^{-r T} \int_{K}^{\infty}\left(S_{T}-K\right) f_{S_{T}}(s) d s
$$

and we should ensure that the first derivative with respect to the strike is negative as follows

$$
\left.\frac{\partial C}{\partial K}\right|_{K=K_{1}}=-e^{-r T} \int_{K_{1}}^{\infty} f_{S_{T}}(s) d s<0
$$

- The last condition is the convexity of the call price $C(K)$ with respect to the strike $K$ If we consider to strikes $K_{1}<K_{2}$ then the first derivative mentionned above should increase in strike

$$
\left.\frac{\partial C}{\partial K}\right|_{K=K_{2}}-\left.\frac{\partial C}{\partial K}\right|_{K=K_{1}}=e^{-r T} \int_{K_{1}}^{K_{2}} f_{S_{T}}(s) d s>0
$$

Defnition 1. A volatility surface is free of static arbitrage if and only if both of the following conditions are satisfied:

1. It is free of calendar spread arbitrage;

2. Each time slice is free of butterfly arbitrage.

Note that the first condition guarantees the monotonicity of European call option prices with respect to theirs maturities. The butterfly arbitrage guarantees the existence of a non negative probability density. Now, we will analyze each condition separately.

\subsubsection{Calendar Spread Arbitrage}

Calendar spread arbitrage refers to the monotonicity of European call option prices with respect to maturity. In order to obtain no arbitrage condition with respect to implied volatility, we need to transform this condition.

To obtain a calendar spread, we will buy and sell options with the same strike price but for different maturities. For simplify, we assume also that there are no dividend or interest rate.

Let $C(T, K)$ the price of a call with expiry $T$ and strike $K$. We know that if $T_{1}<T_{2}$ then we have calendar spread arbitrage if $C\left(T_{1}, K\right)>C\left(T_{2}, K\right)$. 
Our strategy: we buy (long) call option $C\left(T_{2}, K\right)$ (the cheap) and we sell (short) the call $C\left(T_{1}, K\right)$ (the expensive), then, the difference will be $x=C\left(T_{1}, K\right)-C\left(T_{2}, K\right)>0$.

At $T_{1}$ our position is $x+C\left(T_{2}\right)-\max \left\{S_{T_{1}}-K, 0\right\}$

- If $\left(S_{T_{1}} \leq K\right)$, then the total profit is: $x+C\left(T_{2}\right)$

- If $\left(S_{T_{1}}>K\right)$, and the profit will be: $x+C\left(T_{2}\right)-S_{T_{1}}+K$

At $T_{2}$ :

- If $\left(S_{T_{2}}>K\right)$, we buy the stock by paying the amount $K$ that we had received at $T_{1}$, and return the stock that we had short at $T_{1}$. The net profit for our trading strategy is $x>0$.

- If $\left(S_{T_{2}}<K\right)$, we buy the stock by paying $T_{2}$ and return the stock that we short at $T_{1}$. Note that, we had received $k$ at time $T_{1}$, our net profit is then $K-S_{T_{2}}+x>x>0$.

Lemma A volatility surface $w$ is free of calendar spread arbitrage if

$$
\partial_{T} w(k, T) \geq 0, \quad \text { for all } k \in \mathbb{R} \text { and } T>0
$$

Proof : Let $\left(X_{t}\right)_{t \geq 0}$ be a martingale, $L \geq 0$ and $0 \leq t_{1}<t_{2}$. Then the inequality is true

$$
E\left[\left(X_{t_{2}}-L\right)^{+}\right] \geq E\left[\left(X_{t_{1}}-L\right)^{+}\right]
$$

Let consider two options $C_{1}, C_{2}$ with strikes $K_{1}, K_{2}$ and expiry time $t_{1}, t_{2}$ respectively.

If the two options have the same moneyness (log-moneyness or $\log$-strike $:=\log \left(\frac{K}{F_{T}}\right)$ ) we get:

$$
\frac{K_{1}}{F_{t_{1}}}=\frac{K_{2}}{F_{t_{2}}}=: \mathrm{e}^{k}
$$

Then, the process $\left(X_{t}\right)_{t>0}$ defined by $X_{t}:=S_{t} / F_{t}$ is martingale for all $t \geq 0$, and the following relation holds if the dividends are proportional:

$$
\frac{C_{2}}{K_{2}}=\mathrm{e}^{-k} E\left[\left(X_{t_{2}}-\mathrm{e}^{k}\right)^{+}\right] \geq \mathrm{e}^{-k} E\left[\left(X_{t_{1}}-\mathrm{e}^{k}\right)^{+}\right]=\frac{C_{1}}{K_{1}}
$$

This means that if the moneyness is constant, option prices are non-decreasing in time to maturity $\left(\partial_{t} C_{B S}(k, \omega(k, t)) \geq 0\right.$. If this is valid for the Black-Scholes prices $C_{B S}(y, \omega(k, t))$, then $\omega(k, t)$ is strictly increasing, i.e $\left(\partial_{t} w(k, t) \geq 0\right)$.

We can interpret the absence of calender spread by the fact that there are no cross lines on the total variance : when the maturity goes up, the SVI slice will be translated up.

\subsubsection{Butterfly arbitrage}

We consider the butterfly arbitrage for the slice of implied volatility $k \mapsto w(k, t)$.

In the real market the butterfly spread is a strategy with options, which combines simultaneous buying and selling of three similar types options (either calls or puts) with strikes $K-\epsilon<K<K+\epsilon$ and which have the following characteristics:

- The options have the same maturity

- The options are listed on the same underlying

- The strike $K-\epsilon$ and $K+\epsilon$ are equidistant from $K$ as in figure (2.2).

The butterfly consists of the purchase of a call option with strike $K-\epsilon$, the sale of 2 call options with strike $K$ and the purchase of a third option with strike $K+\epsilon$.

In other side, we know that the second derivative of the call price with respect to the strike $k$ is

$$
\frac{d^{2} C(K, T)}{d K^{2}}=\lim _{\varepsilon \rightarrow 0} \frac{C(K-\varepsilon, T)-2 C(K, T)+C(K+\varepsilon, T)}{\varepsilon^{2}}
$$

We consider the following European payoff: 


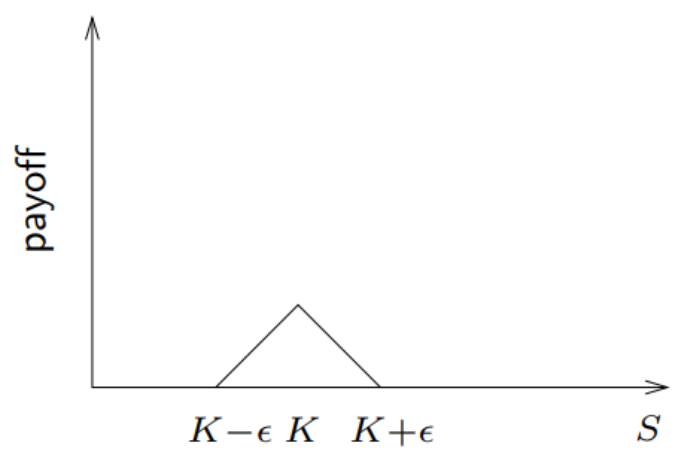

Figure 2.2: Butterfly Spread

- $\frac{1}{\varepsilon^{2}}$ calls with strike $K-\epsilon$

- $\frac{1}{\varepsilon^{2}}$ calls with strikes $K+\epsilon$

- $\frac{-2}{\varepsilon^{2}}$ calls with strikes $k$

This strategy is called the butterfly spread, and the absence of butterfly arbitrage implies that the price of a butterfly spread is positive and the call price must to be convex with

$$
C(K-\epsilon, T)-2 C(K, T)+C(K+\epsilon, T) \geq 0
$$

Let's back to the our SVI model, we will find an equivalent condition to the call price convexity in (2.15) in terms of the implied total variance $w(k)$.

recall the Black-Scholes formula for a European call option price in terms of the total implied variance $w(k)$

$$
C_{\mathrm{BS}}(k, w(k))=S\left(\mathcal{N}\left(d_{+}(k)\right)-\mathrm{e}^{k} \mathcal{N}\left(d_{-}(k)\right)\right), \quad \text { for all } k \in \mathbb{R}
$$

with;

$$
d_{ \pm}(k):=-k / \sqrt{w(k)} \pm \sqrt{w(k)} / 2
$$

Let $P$ be the probability density function of $S_{T}$, then

$$
\begin{gathered}
p(k)=\left.\frac{\partial^{2} C(k)}{\partial K^{2}}\right|_{K=F_{\mathrm{t}} \mathrm{e}^{\mathrm{k}}}=\left.\frac{\partial^{2} C_{\mathrm{BS}}(k, w(k))}{\partial K^{2}}\right|_{K=F_{\mathrm{e}} \mathrm{e}^{\mathrm{k}}}, \quad k \in \mathbb{R} \\
p(k)=\frac{g(k)}{\sqrt{2 \pi w(k)}} \exp \left(-\frac{d_{-}(k)^{2}}{2}\right)
\end{gathered}
$$

Where the function $g: \mathbb{R} \rightarrow \mathbb{R}$

$$
g(k):=\left(1-\frac{k w^{\prime}(k)}{2 w(k)}\right)^{2}-\frac{w^{\prime}(k)^{2}}{4}\left(\frac{1}{w(k)}+\frac{1}{4}\right)+\frac{w^{\prime \prime}(k)}{2}
$$

The condition $g(k)>0$ is equivalent to the market implied volatility density $P(k)$ is positive.

\section{Example: butterfly arbitrage with Calls}

We consider the practical example with three calls options traded on the real market and listed to the same underlying. The three options have the same maturity and strikes: $k_{1}=10, k_{2}=20$ and $k_{3}=30$.

The three options are quoted as following:

$$
\operatorname{Call}\left(k_{1}=10\right)=12 \$, \quad \operatorname{Call}\left(k_{1}=20\right)=7 \$, \quad \operatorname{Call}\left(k_{1}=30\right)=1 \$
$$

The question: is there any arbitrage opportunity?

Response: As we know that the call option price is a convex function with respect to the strike. Let's consider $C(K)$, the call price function with strike $\mathrm{K}$, and we have only to check the convexity criterion using (2.3.3). 
The $C(K)$ is convex if and only if

$$
C\left(\frac{K_{1}+K_{3}}{2}\right) \leq \frac{1}{2}\left(C\left(K_{1}\right)+C\left(K_{3}\right)\right)
$$

This is equivalent to check the inequality

$$
C(20) \leq \frac{1}{2}(C(10)+C(30))=6.5
$$

However, $C(20)=7$ and the inequality is not satisfied, therefore, there is a butterfly arbitrage opportunity.

In this case, our arbitrage strategy is to take advantage (profit) in this situation: we short (i.e., sell) the options that are over-priced (i.e $\operatorname{Call}\left(k_{1}=20\right)=7$ ), and we long (i.e., buy) the options that are under-priced (i.e $\operatorname{Call}\left(k_{1}=10\right)=12 \$$ and $\operatorname{Call}\left(k_{1}=30\right)=1 \$$ ).

We short two calls options $\operatorname{Call}\left(k_{1}=20\right)$ and long the two others calls $\operatorname{Call}\left(k_{1}=10\right) \$$ and $\operatorname{Call}\left(k_{1}=30\right) \$$

The profit at the present time $t$ is : $2 \times 7-12-1=1 \$$.

At maturity the pay-off will be always positive as below

$$
\left(S_{T}-10\right)^{+}+\left(S_{T}-30\right)^{+}-2\left(S_{T}-20\right)^{+}= \begin{cases}0, & \text { if } S_{T} \leq 10 \\ S_{T}-10, & \text { if } 10 \leq S_{T} \leq 20 \\ 30-S_{T}, & \text { if } 20 \leq S_{T} \leq 30 \\ 0, & \text { if } S_{T} \geq 30\end{cases}
$$

We note that we have guaranteed profit at the start time $t$ and also at maturity $T$.

Note that we can also use another method to verify the butterfly arbitrage. In fact, we have no butterfly arbitrage in our input data if and only if the slope is decreasing in the call function $C(k)$ which is can be written as

$$
\frac{C_{i+1}-C_{i}}{K_{i+1}-K_{i}} \geq \frac{C_{i}-C_{i-1}}{K_{i}-K_{i-1}}
$$

If the slope increasing, this mean that $C(k)$ is convex. We use this to check in our example

$$
\begin{gathered}
\operatorname{Call}\left(k_{1}=10\right)=12 \$, \quad \operatorname{Call}\left(k_{2}=20\right)=7 \$, \quad \operatorname{Call}\left(k_{3}=30\right)=1 \$ \\
\frac{1-7}{30-20} \geq \frac{7-12}{20-10}
\end{gathered}
$$

We get

$$
\frac{-6}{10} \geq \frac{-5}{10}
$$

The inequality is not verified, hence, there is a butterfly arbitrage.

\section{Example: Butterfly arbitrage with two Puts}

In order to construct a butterfly arbitrage, we will use in this example two European puts with non-dividend and the following characteristics:

$$
\operatorname{Put}\left(k_{2}=80\right)=8 \$ \quad \operatorname{Put}\left(k_{3}=90\right)=9 \$
$$

The same question as with the previous example: is there any arbitrage opportunity?

Response: Let's consider Put $(K)$, the put price function with strike K, and we can check the convexity criterion using (2.26). In fact, we need a third point to calculate the convexity criterion, hence, and we consider the three points

$$
K_{1}=0, \quad K_{2}=80, \quad K_{3}=90
$$

Where $\operatorname{Put}\left(k_{1}=0\right)=0 \$$, then we can write

$$
K_{2}=1 / 9 K_{1}+8 / 9 K_{3}
$$


The function $\operatorname{Put}(K)$ is convex if and only if

$$
\begin{aligned}
\operatorname{Put}\left(K_{2}\right) & =\operatorname{Put}\left(\frac{1}{9} K_{1}+\frac{8}{9} K_{3}\right) \\
& \leq \frac{1}{9} \operatorname{Put}\left(K_{1}\right)+\frac{8}{9} \operatorname{Put}\left(K_{3}\right) \\
& =\frac{8}{9} \operatorname{Put}\left(K_{3}\right)
\end{aligned}
$$

Therefore, the inequality is satisfied, and the put in convex, hence, there are no arbitrage in this example.

Defnition 2.1 A slice is said to be free of buttery arbitrage if the corresponding density define in (2.19) is non-negative, which is equivalent to $g(k) \geq 0$.

Lemma 2.2 The map $k \mapsto w(k, t)$ is free arbitrage if and only if $g(k) \geq 0$ for all $k \in \mathbb{R}$ and $\lim _{t \rightarrow+\infty} d_{+}(k)=-\infty$

Proof: Let $P$ the probability density function that can be calculated from the call price function $C(k)$ using the formula of Breeden-Litzemberger is:

$$
p(k)=\left.\frac{\partial^{2} C(k)}{\partial K^{2}}\right|_{K=F_{t} \mathrm{e}^{k}}=\left.\frac{\partial^{2} C_{\mathrm{BS}}(k, w(k))}{\partial K^{2}}\right|_{K=F_{t} \mathrm{e}^{k}}, \quad k \in \mathbb{R}
$$

By diferentiating the Black-Scholes formula for any $k \in \mathbb{R}$, we get ;

$$
p(k)=\frac{g(k)}{\sqrt{2 \pi w(k)}} \exp \left(-\frac{d_{-}(k)^{2}}{2}\right)
$$

We note that the integral of the density function may not all time equal to 1 , and we need to impose asymptotic boundary conditions. The limit of the call option $\lim _{k \rightarrow+\infty} C_{\mathrm{BS}}(k, w(k))=0$, which is equivalent to $\lim _{k \rightarrow+\infty} d_{+}(k)=-\infty$.

Now we will summarize the characterisation of static arbitrage that we will use in practice in the next section of calibration.

\begin{tabular}{llll}
\multicolumn{1}{c}{ AOA Characterisation } & Call criterion & equivalent criterion & equivalent criterion \\
\hline Butterfly arbitrage (Convexity) & $\left.\frac{\partial^{2} C(k)}{\partial K^{2}}\right|_{K=F_{t} e^{k}} \geq 0$ & $p(k) \geq 0$ & $g(k) \geq 0$ \\
Limit Price function & $\lim _{K \rightarrow+\infty} C_{\mathrm{BS}}(T, k)=0$ & $\lim _{t \rightarrow+\infty} d_{+}(k)=-\infty$ & $b(1+\rho)<2$ \\
Calendar Spread (Monotonicity) & $\mathrm{T} \longrightarrow C_{\mathrm{BS}}(T, K) \nearrow$ & $\frac{\partial C_{\mathrm{BS}}(T, x)}{\partial T} \geq 0$ & $\partial_{T} w(k, T) \geq 0$
\end{tabular}

Table 2.1: Summary of the static arbitrage 
Axel Vogt example of arbitrage: generated by a parametrisation of SVI with $\mathrm{T}=1$

$$
(a, b, m, \rho, \sigma)=(-0.0410,0.1331,0.3586,0.3060,0.4153)
$$

These parameters give a positive total variance function $w$, however, the density function $p(k)$ and $g(k)$ defined in (3.20), are negative. We will show explicitly, in the next section, how to tackle this problem.
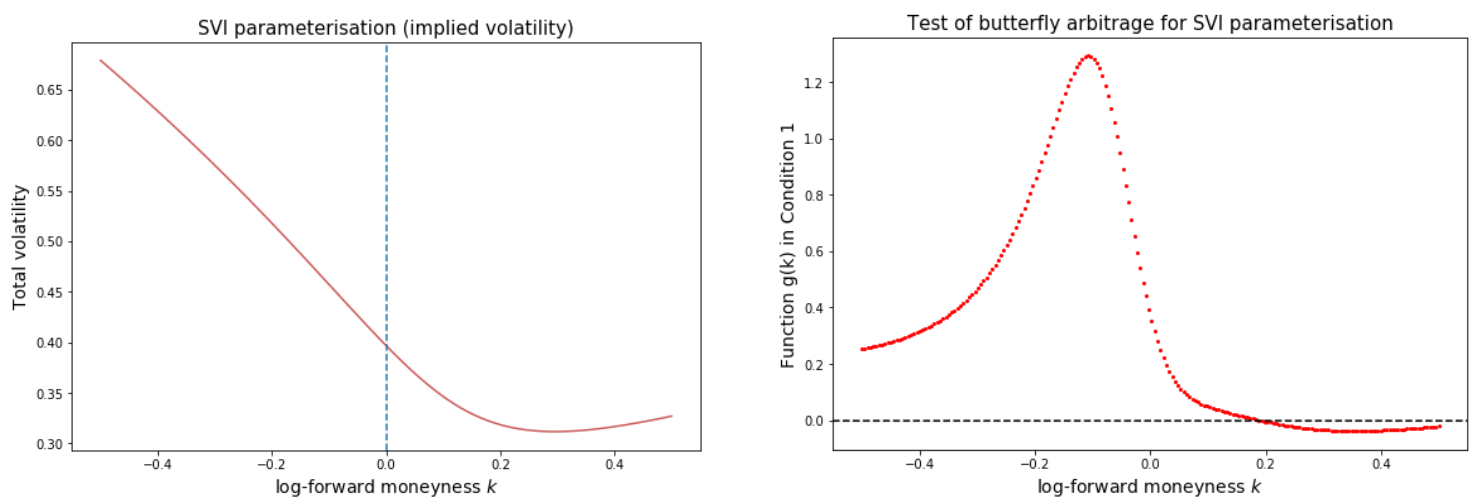

Figure 2.3: Plots of the total variance smile $w$ (left) and the function $g$ (right), using Axel Vogt's parameters

\subsection{Volatility on the wings}

Inspired by the work done by Jäckel in [18], in this section In this section we will proceed to an asymptotic study of the function $g(k)$, for large strikes, in order to get sufficient conditions that ensure a probability density function $P(k)$ positive and hence, an SVI butterfly arbitrage free.

We recall the $g: \mathbb{R} \rightarrow \mathbb{R}$

$$
g(k):=\left(1-\frac{k w^{\prime}(k)}{2 w(k)}\right)^{2}-\frac{w^{\prime}(k)^{2}}{4}\left(\frac{1}{w(k)}+\frac{1}{4}\right)+\frac{w^{\prime \prime}(k)}{2}
$$

In the wings we know that the total implied variance $w\left(k ; \chi_{N}\right)$ has the left and right asymptotes that respect the assumption of linear wings, this result is consistent with the Roger Lee's moment formula mentioned above.

$$
\left\{\begin{array}{lll}
W_{R}(k)=b(\rho+1) k+[a-b m(\rho+1)] & k \rightarrow+\infty \\
W_{L}(k)=b(\rho-1) k+[a-b m(\rho-1)] & k \rightarrow-\infty
\end{array}\right.
$$

In the general case (left and right wing), we consider the total implied variance $w\left(k ; \chi_{N}\right)$ for large strikes.

$$
w(k)=\alpha+\beta k, \quad \text { and } \quad w^{\prime}(k)=\beta, \quad w^{\prime \prime}(k)=0
$$

We replace in (2.32) and we get

$$
g(k)=\left(1-\frac{k}{2} \frac{\beta}{(\alpha+\beta k)}\right)^{2}-\frac{\beta^{2}}{4}\left(\frac{1}{\alpha+\beta k}+\frac{1}{4}\right)
$$

After simplification we get the following quadratic equation

$$
g(k)=\frac{\beta^{2}}{4}\left(1-\frac{\beta^{2}}{4}\right) k^{2}+\frac{1}{4}\left(4 \alpha \beta-\beta^{3}-\frac{\alpha \beta^{3}}{2}\right) k+\frac{1}{4}\left(4 \alpha^{2}-\alpha \beta^{2}-\frac{\alpha^{2} \beta^{2}}{4}\right)
$$

We calculate the discriminant $\Delta$ of the quadratic function $g(k)$ and we get

$$
\Delta=\frac{\beta^{4}}{16}\left(\beta^{2}+\alpha^{2}-4 \alpha\right)
$$


In order to guarantee that the function $g(k)$ is positive, we will be interested to the case where the discriminant $\Delta$ of the quadratic function $g(k)$ is strictly negative in both wings sides $(\Delta<0)$ and the first coefficient of the $k^{2}$ is strictly positive also.

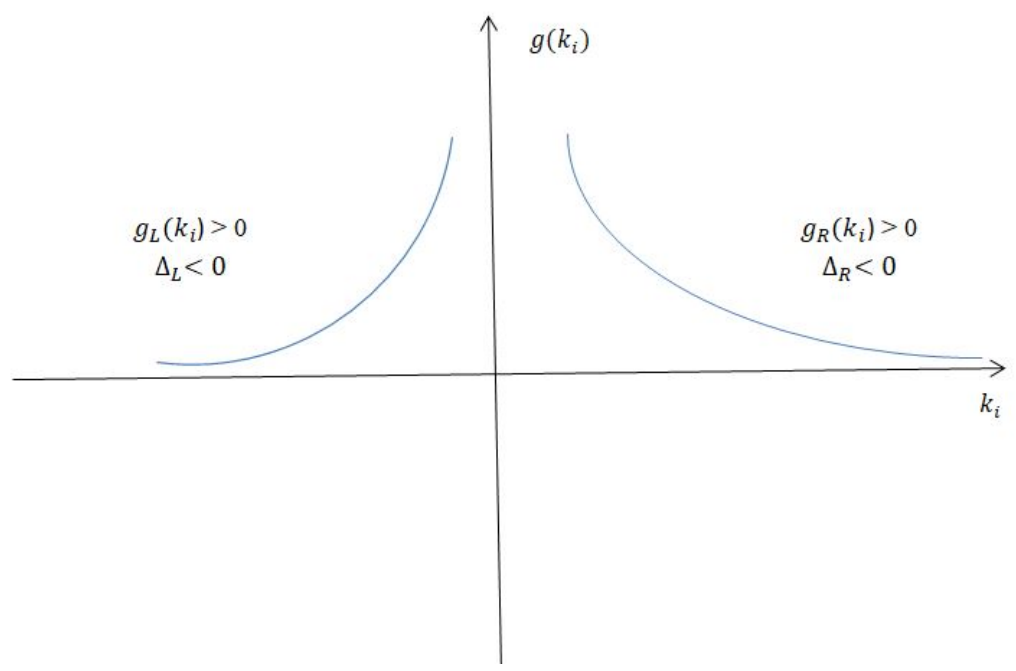

Figure 2.4: Large strikes asymptotic for the $g(k)$ function

We study both wings, right and left, to find sufficient conditions for the butterfly arbitrage.

\subsubsection{The right wing}

We avoid the arbitrage in the right wing by considering that the discriminant $\Delta_{R}$ is negative in the right side of the function $g(k)$.

We recall the asymptotic formula, for large strikes, for the total implied variance

$$
W_{R}(k)=b(\rho+1) k+[a-b m(\rho+1)]=\alpha+\beta k \quad k \rightarrow+\infty
$$

Where;

$$
\alpha=a-b m(\rho+1), \quad \beta=b(\rho+1)
$$

The discriminant $\Delta_{R}$ is calculated by replacing $\alpha$ and $\beta$ in (2.36)

$$
\Delta_{R}=\frac{b^{4}(\rho+1)^{4}}{16}\left[b^{2}(\rho+1)^{2}+(a-m b(\rho+1))^{2}-4(a-b m(\rho+1))\right]
$$

As the term $\frac{b^{4}(\rho+1)^{4}}{16}$ is strictly positive, the sign of $\Delta_{R}$ will be decided by the second term, and we get

$$
\begin{aligned}
\left(\Delta_{R}<0\right) & \Longleftrightarrow\left(b^{2}(\rho+1)^{2}+(a-m b(\rho+1))^{2}-4(a-b m(\rho+1))<0\right) \\
& \Longleftrightarrow b^{2}(\rho+1)^{2}<(a-m b(\rho+1))(4-a+b m(\rho+1)) \\
& \Longleftrightarrow \frac{(a-m b(\rho+1))(4-a+b m(\rho+1))}{b^{2}(\rho+1)^{2}}>1
\end{aligned}
$$

Where; $b^{2}(\rho+1)^{2}>0$

$\Delta_{R}$ is negative, hence, the quadratic function $g(k)$ is strictly positive if the first coefficient of this quadratic function is positive, i.e

$$
\begin{aligned}
\frac{\beta^{2}}{4}\left(1-\frac{\beta^{2}}{4}\right)>0 & \Longleftrightarrow \frac{b^{2}(\rho+1)^{2}}{4}\left(1-\frac{b^{2}(\rho+1)^{2}}{4}\right)>0 \\
& \Longleftrightarrow 0<b^{2}(\rho+1)^{2}<4
\end{aligned}
$$


Finally, for the right wing, $g_{R}(k)$ is strictly positive if the following two conditions satisfies

$$
\left\{\begin{array}{r}
\frac{(a-m b(\rho+1))(4-a+b m(\rho+1))}{b^{2}(\rho+1)^{2}}>1 \\
0<b^{2}(\rho+1)^{2}<4
\end{array}\right.
$$

\subsubsection{The left wing}

We fellow the same analysis as the previous one, the calculus is the same we change only the term $(\rho+1)$ by $(\rho-1)$.

We can avoid the arbitrage in the left wing by considering that the discriminant $\Delta_{L}$ is negative in the left side of the function $g_{L}(k)$.

We recall the total implied variance left asymptotic formula for the large strikes

$$
W_{L}(k)=b(\rho-1) k+[a-b m(\rho-1)]=\alpha+\beta k \quad k \rightarrow+\infty
$$

Where;

$$
\alpha=a-b m(\rho-1), \quad \beta=b(\rho-1)
$$

The discriminant $\Delta_{L}$ is calculated by replacing $\alpha$ and $\beta$ in (2.36)

$$
\Delta_{R}=\frac{b^{4}(\rho-1)^{4}}{16}\left[b^{2}(\rho-1)^{2}+(a-m b(\rho-1))^{2}-4(a-b m(\rho-1))\right]
$$

As the term $\frac{b^{4}(\rho-1)^{4}}{16}$ is strictly positive, the sign of $\Delta_{L}$ will be decided by the second term, and we get

$$
\begin{aligned}
\left(\Delta_{L}<0\right) & \Longleftrightarrow\left(b^{2}(\rho-1)^{2}+(a-m b(\rho-1))^{2}-4(a-b m(\rho-1))<0\right) \\
& \Longleftrightarrow b^{2}(\rho-1)^{2}<(a-m b(\rho-1))(4-a+b m(\rho-1)) \\
& \Longleftrightarrow \frac{(a-m b(\rho-1))(4-a+b m(\rho-1))}{b^{2}(\rho-1)^{2}}>1
\end{aligned}
$$

Where; $b^{2}(\rho-1)^{2}>0$

$\Delta_{L}$ is negative, hence, the quadratic function $g_{L}(k)$ is strictly positive if the first coefficient of this quadratic function is positive, i.e

$$
\begin{aligned}
\frac{\beta^{2}}{4}\left(1-\frac{\beta^{2}}{4}\right)>0 & \Longleftrightarrow \frac{b^{2}(\rho-1)^{2}}{4}\left(1-\frac{b^{2}(\rho-1)^{2}}{4}\right)>0 \\
& \Longleftrightarrow 0<b^{2}(\rho-1)^{2}<4
\end{aligned}
$$

Finally, for the right wing, $g_{R}(k)$ is strictly positive if the following two conditions are satisfied

$$
\left\{\begin{array}{r}
\frac{(a-m b(\rho-1))(4-a+b m(\rho-1))}{b^{2}(\rho-1)^{2}}>1 \\
0<b^{2}(\rho-1)^{2}<4
\end{array}\right.
$$

We summary the necessary conditions that guarantee a butterfly arbitrage free for SVI model as following 


\section{Theorem 2.2}

The total implied variance $w(k, t)$ in the SVI model is said to be free of butterfly arbitrage if the following conditions holds

$$
\left\{\begin{array}{r}
\frac{(a-m b(\rho+1))(4-a+b m(\rho+1))}{b^{2}(\rho+1)^{2}}>1 \\
\frac{(a-m b(\rho-1))(4-a+b m(\rho-1))}{b^{2}(\rho-1)^{2}}>1 \\
0<b^{2}(\rho+1)^{2}<4 \\
0<b^{2}(\rho-1)^{2}<4
\end{array}\right.
$$




\section{Chapter 3}

\section{Robust Calibration For SVI Arbitrage Free}

In this chapter we present our main result: robust calibration that produces an arbitrage free set of raw SVI parameters. One of the desirable features of the SVI model is that it fits the input data very well especially in the equity market.The main weakness of this model is that arbitragefreeness in strike and maturity is not guarantee and it's not automatically verified during the calibration step. More often, we calibrate the model and next we check the arbitrage condition via the risk neutral density(RND) if it's positive. In practice, this is a quiet inconvenience and most practitioners require a more robust approach.

We implement and test a robust calibration method using the Sequential Quadratic Programming (SQP) optimization method with constraints that allow to calibrate the SVI model and also to eliminate the arbitrage automatically during the calibration step. We no longueur need to check the positivity of the density function $p(k)$. This calibration method is very important in practice: it allows to implement the model in the pricing library and also to calculate the local volatility using the implied volatility. Finally, we give two examples with arbitrage (one of them is Axel Vogt) and we show in practice how our algorithm could perform in this case.

Finally, we test the performance of our calibration method using the implied volatility extracted from call/put option price listed in 23 indexes, each one with 14 maturities.

\subsection{The Raw SVI Calibration}

The raw SVI model represents the total variance or the implied volatility of the call or put options observed in the market. Therefore, we define a loss function to be optimized. This function is the difference between the value of variance given by our SVI model and the value of implied volatility observed in the market. The minimization of this function will permit us to calibrate SVI to observed implied volatilities.

Most algorithms require an initial guess of the model parameters, as well as boundaries conditions and an objective function. The resulting parameters should respect the arbitrage free conditions: butterfly arbitrage and calendar spread arbitrage.

Recall the expression of the raw SVI model with 5 parameters $\chi_{R}=\{a, b, \rho, m, \sigma\}$.

$$
w_{\text {Total }}\left(k ; \chi_{R}\right)=a+b\left\{\rho(k-m)+\sqrt{(k-m)^{2}+\sigma^{2}}\right\}
$$

For every strike $k \in \mathbb{R}$, and

$$
\begin{aligned}
a & \in \mathbb{R} \\
b & \geq 0 \\
|\rho| & <1 \\
m & \in \mathbb{R} \\
\sigma & >0 \\
a+b \sigma \sqrt{1-\rho^{2}} & \geq 0
\end{aligned}
$$




\subsection{SVI's Parameters Boundaries}

The purpose of this section is to determine lower and upper boundaries of each of the SVI parameters $(a, b, \rho, m, \sigma)$ in order to run an efficient calibration that respect conditions related to the model and also to avoid arbitrage.

We have some restrictions on the parameters that follow from the parameterization of the model in (2.1), such as:

$$
b \geq 0 ; \quad|\rho|<1 ; \quad \sigma>0 .
$$

Zeliade [19] present some parameter constraints and limiting cases. They incorporate also some boundaries that are deduced the no arbitrage conditions. We present them bellow.

\section{- Parameter $a$ and SVI Minimum}

In this part we define the lower and upper bound of the parameter $a$.

$w\left(k ; \chi_{R}\right)$ has a unique minimum if $\rho^{2} \neq 1$ and it's value is

$$
W_{\min }(k)=a+b \sigma \sqrt{1-\rho^{2}}
$$

This minimum is located at the point: $k^{*}=m-\frac{\rho \sigma}{\sqrt{1-\rho^{2}}}$.

We replace the positivity condition: $a+b \sigma \sqrt{1-\rho^{2}}>0$ by the restriction $a>0$

which is stronger as both parameters $b$ and $\sigma$ are positive and obviously $\sqrt{1-\rho^{2}}$ also. Finally, we can conclude that the conditions

$$
W_{\min }(k)=a+b \sigma \sqrt{1-\rho^{2}}>0 \Longleftrightarrow(a>0)
$$

If we impose the condition $a>0$, the question came up: which lower bound could $a$ take. This is a very important question that we will discuss it in the calibration section.

At present we take an arbitrary small value $a_{\min }=10^{-5}$.

For the upper bound, and as the parameter a represents the overall level of total variance or the vertical translation, hence, it could not suitable to get a value of $a$ greater than the largest value of the observed total variance. Therefore we impose for $a$ the following boundaries

$$
0<a_{\min }=10^{-5} \leq a \leq \max \left(W_{S V I}^{\operatorname{market}}\right)
$$

- Parameter $b$ and left wing

we can estimate the left and the right SVI asymptotes:

$$
\begin{aligned}
& W_{L}(k)=a+b(\rho-1)(k-m) \\
& W_{R}(k)=a+b(\rho+1)(k-m)
\end{aligned}
$$

For the right wing the slop is $b(\rho+1)$ and it should not exceed to 2 which is consistent with Roger Lee formula value of implied volatility for the large strike tail [13].

We can also take the advantage from the others arbitrage constraints such as

$$
\lim _{K \rightarrow+\infty} C_{\mathrm{BS}}(T, k)=0 \Longleftrightarrow \lim _{t \rightarrow+\infty} d_{+}(k)=-\infty
$$

We show that the condition $\lim _{k \rightarrow \infty} d_{1}(k, w(k))=-\infty$, i.e :

$$
\lim _{k \rightarrow \infty} d_{1}\left(k, w_{\mathrm{SVI}}(k)\right)=\lim _{k \rightarrow \infty}\left(-\frac{k}{\sqrt{w_{\mathrm{SVI}}(k)}}+\frac{1}{2} \sqrt{w_{\mathrm{SVI}}(k)}\right)=-\infty
$$

Is satisfied for a function $w(k)$ if:

$$
\limsup _{k \rightarrow \infty} \frac{w(k)}{2 k}<1 .
$$


Or we have :

$$
\limsup _{k \rightarrow \infty} \frac{w(k)}{k}=b(\rho+1)
$$

Finally, to be consistent with the Roger Lee's moment formula, the right slope condition should satisfy the inequality:

$$
b(\rho+1)<2 .
$$

Using this last inequality we can conclude that for $\rho \neq-1$

$$
b<\frac{2}{(\rho+1)}
$$

For any $|\rho|<1$, the minimum of the function $f(\rho)=\frac{2}{(\rho+1)}$ is $f(1)=1$, (with $\left.|\rho|<1\right)$.

For the $b$ lower bound, we can take a small value around $b_{\min }=10^{-2}$.

Finally we obtain that the $b$ boundaries are:

$$
0<b_{\text {min }}=10^{-2}<b<1
$$

\section{- Correlation Parameter $\rho$}

The correlation coefficient between the Brownian motion of the underlying and the implied variance process is $\rho$, hence, it's value will be in the interval ] - 1,1[

\section{- Translation Parameter $m$}

By the same way, $m$ is the translation of the smile to the right, and as the smile could not more out side the zone limited by the log forward moneyness of our input data, hence, we can cap it at some reasonably translation level as following.

$$
2 \min _{i} k_{i} \leq m \leq 2 \max _{i} k_{i}
$$

Where $k_{i}$ is the $\log$ forward moneyness or $\log$ strike: $k_{i}:=\log \left(\frac{K_{i}}{F_{T}}\right)$.

\section{- Curvature Parameter $\sigma$}

The positive value of sigma $(\sigma>0)$ means that the total implied variance has a positive at-the-money curvature.

As $\sigma$ represents the curvature, it's rare to see $\sigma$ takes values larger than 1: in general, $\sigma$ takes small values around 0.01 or 0.02 for the short maturities and it goes more large for long maturities, like 0.2 or 0.3 for 10 years time to expiry.

For this reason, we can cap sigma by any arbitrary reasonable value $\sigma_{\max }=$ constant value such as 1,2 or other number.

$$
0<\sigma_{\min }=0.01 \leq \sigma \leq \sigma_{\max }=1
$$

We can summarize the obvious boundaries for the SVI raw parameters model as following:

$$
\left\{\begin{array}{r}
0<a_{\min }=10^{-5} \leq a \leq \max \left(W_{S V I}\right) \\
0<b_{\min }=0.001<b<1 \\
-1<\rho<1 \\
2 \min _{i} k_{i} \leq m \leq 2 \max _{i} k_{i} \\
0<\sigma_{\min }=0.01 \leq \sigma \leq \sigma_{\max }=1
\end{array}\right.
$$

The boundaries presented in (3.72) are very important to satisfy during the calibration, respecting these values will allow us to take an advantage and to avoid some arbitrage zones. The last point to care about is the butterfly arbitrage related to guarantee that the probability density function is positive, this is what we will see in the next section. 


\subsubsection{The Initial Guess}

In order to run our calibration algorithm, we need to define the initial guess parameter's values, this values are very important and we should to care when we choose these right values. Also, the initial point should below to the boundaries intervals for each variable.

For the first parameter $a$ that represent the vertical translation, one of the reasonable value is to be close to the minimum of the market total variance value, hence, we can take it

$$
a=\frac{1}{2} \min \left(W_{S V I}\right)
$$

The parameter $b$ could be taken as $b=0.1$, in general this value is the middle between the $b$ value for the short maturities of one weeks to the large maturities of 10 years.

For the correlation parameter $\rho$, it's obvious to guess it's initial value for the equity market, we are sure that it takes negative value and the initial guess should be

$$
\rho=-0.5
$$

Note that the extreme values of $\rho$ are -1 and 1 , for $\rho=1$, the SVI model is graphically similar to the payoff of the call, and for $\rho=-1$, the $\rho=-1$, the SVI model ( the smile) is similar to the put payoff.

Therefore $\rho=-0.5$, is a reasonable value for the smile in the equity market. We will see after calibration that $\rho$ vary with respect to the time to expiry: more the time to expiry is large, more will decrease taking small negative values.

Regarding the horizontal translation parameter $m$ : we notice that $m$ can take as positive or negative values: more the $m$ value is high, more the smile will be translated to the right and vice versa. The initial value $m=0.1$, is a resealable value between the short and long maturities.

The last parameter is the curvature parameter $\sigma$ which is strictly positive in general it's values are small around 0.01 for the short maturities ( weekly or monthly) and 0.2 for the large maturities of 10 years, hence, taking $\sigma=0.1$ will be acceptable value valid for the short and the long maturities.

Note that these values are arbitrary and any other values close could be work also, finally, we summarize the initial guess values for the SVI calibration in the box bellow.l

$$
\left\{\begin{aligned}
a=\frac{1}{2} \min \left(W_{S V I}\right) \\
b=0.1 \\
\rho=-0.5 \\
m=0.1 \\
\sigma=0.1
\end{aligned}\right.
$$

\subsubsection{The Butterfly Arbitrage Constraints}

Let's recall the expression of $P$, the probability density function that can be calculated from the call price function $C(k)$ using the formula of Breeden-Litzemberger [11]:

$$
p(k)=\left.\frac{\partial^{2} C(k)}{\partial K^{2}}\right|_{K=F_{t} \mathrm{e}^{k}}=\left.\frac{\partial^{2} C_{\mathrm{BS}}(k, w(k))}{\partial K^{2}}\right|_{K=F_{t} \mathrm{e}^{k}}, \quad k \in \mathbb{R}
$$

By diferentiating the Black-Scholes formula for any $k \in \mathbb{R}$, we get ;

$$
p(k)=\frac{g(k)}{\sqrt{2 \pi w(k)}} \exp \left(-\frac{d_{-}(k)^{2}}{2}\right)
$$

The limit of the call option $\lim _{k \rightarrow+\infty} C_{\mathrm{BS}}(k, w(k))=0$, which is equivalent to:

$$
\lim _{k \rightarrow+\infty} d_{+}(k)=-\infty
$$

Our model is butterfly arbitrage free if the SVI parameters could guarantee that the probability density function $P$ is positive for any log forwad moneyness $k$, hence, $p(k)$ is positive if and only if 
the function is also positive $(g(k)>0)$.

Where the function $g: \mathbb{R} \rightarrow \mathbb{R}$

$$
g(k):=\left(1-\frac{k w^{\prime}(k)}{2 w(k)}\right)^{2}-\frac{w^{\prime}(k)^{2}}{4}\left(\frac{1}{w(k)}+\frac{1}{4}\right)+\frac{w^{\prime \prime}(k)}{2}
$$

Finally, we can summarize all the constraints that could guarantee to get SVI model with arbitrage free following these conditions:

$$
\left\{\begin{array}{r}
0<a_{\text {min }}=10^{-5} \leq a \leq \max \left(W_{S V I}\right) \\
0<b_{\text {min }}=0.001 \leq b<1 \\
-1<\rho<1 \\
2 \min _{i} k_{i} \leq m \leq 2 \max _{i} k_{i} \\
0<\sigma_{\text {min }}=0.01 \leq \sigma \leq \sigma_{\max }=1 \\
g(k)>0
\end{array}\right.
$$

\subsection{Input Data}

We download Market data for example $\operatorname{Call}_{\mathrm{BS}}(k, w(k))$ or $\operatorname{Put}_{\mathrm{BS}}(k, w(k))$, for an expiry time T fix with strikes $K$, and next we can extract the implied volatility from this market call or put prices by inverting the black Scholes formula, finally, we get what we call the implied variance $\sigma_{B S}^{2}(k, T)$.

We calculate the total implied variance : $\omega_{\text {Total }(i)}^{\text {market }}=T \sigma_{B S}^{2}(k, T)$

Calculate the $\log$ forward moneyness or $\log$ strike: $x:=\log \left(\frac{K}{F_{T}}\right)$, where $F_{T}$ is the forward price. $^{1}$

Our data is the prices of index options Call and Put listed on 23 indexes such as: EURO STOXX 50, CAC 40, NIKKEI 225, FTSE Mid 250 Index, SWISS MARKET IND, Hang Seng, NASDAQ 100, FTSE 100, MSCI world TR Index, Sao Paulo SE Bovespa Index,...etc

We display a sample of data call and put prices listed in the EURO STOXX 50 index in the figure $(3.1)$.

\begin{tabular}{cccc} 
Strikes & Implied volatility $\%$ & Call & Put \\
\hline 2068.48 & 24.93 & 1268.59 & 7.25 \\
2413.23 & 22.3 & 936.83 & 21.56 \\
2757.98 & 19.39 & 621.99 & 52.79 \\
3016.54 & 17.17 & 407.05 & 97.39 \\
3585.37 & 12.86 & 77.18 & 338.53 \\
3964.59 & 12.22 & 15.1 & 657.11 \\
4481.71 & 12.98 & 1.87 & 1162.98 \\
4998.83 & 14.17 & 0.35 & 1680.55 \\
5688.33 & 15.65 & 0.05 & 2372.38 \\
6033.07 & 16.32 & 0.02 & 2718.41 \\
6377.82 & 16.96 & 0.01 & 3064.46 \\
6722.57 & 17.56 & 0.01 & 3410.52 \\
6894.94 & 17.86 & 0 & 3583.55 \\
\hline
\end{tabular}

Table 3.1: Call and Put Price on 2019 /04/05 with, T =1.01 Y, from EURO STOXX 50

We can note in the figure (3.1) that our data respect some proprieties such positivity of both prices call and put and secondly monotonicity: put options price are increasing, and call options price

\footnotetext{
${ }^{1}$ Some times the strike is a large number, to reduce this strike scale, we should use this variable change to the logarithmic scale in order to get a small numbers in the $\mathrm{x}$-axis.
} 


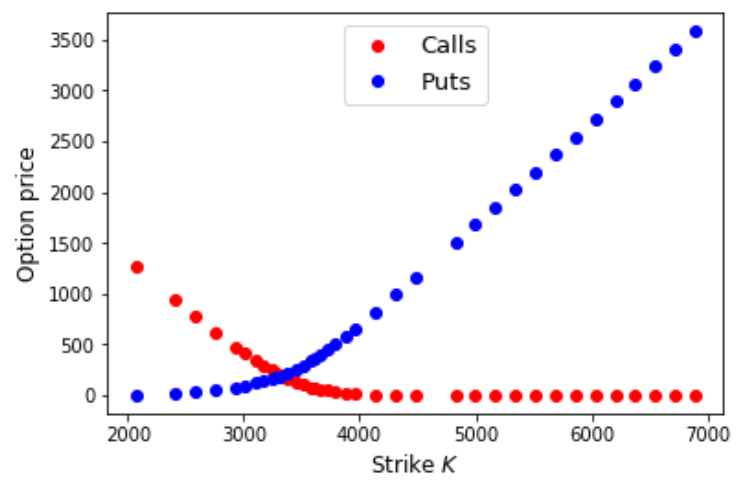

Figure 3.1: Call and Put option prices from Eurostoxx50, (T : 2019/04/05 to 2020/04/06 )

are decreasing with respect to the strike. These proprieties are the basic requirements that our data should respect. Note also that we can check the convexity of our option prices ensuring that the second derivative is positive point by point.

\subsection{Sequential Quadratic Programming For SVI Calibration}

Sequential Least-Squares Quadratic Programming (SLSQP) is a sequential quadratic programming (SQP) algorithm for nonlinearly constrained gradient-based optimization. Sequential quadratic programming method SQP is proposed for the first time by Wilson in his PhD thesis (1963).

This method is used for constrained nonlinear optimization problems with any combination of bounds. The idea behind this method is to reformulate and to solve the original function to a sub-problem in each iteration by linearization of the constraints and approximating the Lagrangian function.

This method is the exact solution to our problem which is a non linear optimization with non linear constraints. First, we present an overview of this method in the general case as presented by Dieter Kraft in [20].

First, we start by defining the convergence rates of sequence as define in [21].

Definition 4.4 We consider $\left(x^{k}\right)_{k \in N_{0}}$ a sequence of iterates converging to $x^{*}$, the sequence is said to convergence

- Linearly, if there exist $0<q<1$ and $k_{\max } \geq 0$ such that for all $k \geq k$

$$
\left\|x^{k+1}-x^{*}\right\| \leq q\left\|x^{k}-x^{*}\right\|
$$

- Superlinearly, if there exist a null sequence $\left(q_{k}\right)_{k \in N_{0}}$ of positive numbers and $k_{\max } \geq 0$ such that for all $k \geq k_{\max }$

$$
\left\|x^{k+1}-x^{*}\right\| \leq q_{k}\left\|x^{k}-x^{*}\right\|
$$

- Quadratically, if there exist $c>0$ and $k_{\max } \geq 0$ such that for all $k \geq k_{\max }$

$$
\left\|x^{k+1}-x^{*}\right\| \leq c\left\|x^{k}-x^{*}\right\|^{2}
$$

- R-linearly, if there exist $0<q<1$ such that

$$
\limsup _{k \rightarrow \infty} \sqrt[k]{\left\|x^{k}-x^{*}\right\|} \leq \sqrt[k]{q}
$$




\subsubsection{The Nonlinear Programming Problem NLP}

A nonlinear programming problem is the minimization of non linear objective function $f(x)$, $\mathbf{x} \in \mathbb{R}^{n}$ with $n$ variables subject to equation or inequality constraints, and the general form is as following.

\section{The Non Linear Problem (NLP)}

$$
(\mathbf{N L P}): \min _{x \in R^{n}} f(x)
$$

Subject to

$$
\begin{gathered}
g_{j}(x)=0, \quad j=1, \ldots, m_{c} \\
g_{j}(x) \geq 0, \quad j=m_{e}+1, \ldots, m \\
x_{l} \leq x \leq x_{n}
\end{gathered}
$$

Where the functions $f: R^{n} \rightarrow R^{1}$ and $g: R^{n} \rightarrow R^{m}$ are continuously differentiable and don't have a specific structure.

The solution of the (NLP) problem is iteratively: we start with initial value of the parameter's vector $x^{0}$, and the $(\mathrm{k}+1)$ interation of $x^{k+1}$ will be obtained from the previous one of $x^{k}$.

$$
x^{k+1}:=x^{k}+\alpha^{k} d^{k}
$$

Where $d^{k}$ is the search direction in the $k^{\text {th }}$ step and $\alpha^{\text {th }}$ is the step length.

\section{The Search Direction}

To find the search direction $d^{k}$, we reformulate the original (NLP) problem to a quadratic programming subproblem by a quadratic approximation of the Lagrange function of the (NLP) problem and a linear approximation of the constraints function $g_{j}$.

The Lagrange function of the (NLP) problem is defined by

$$
L(x, \lambda)=f(x)-\sum_{j=1}^{m} \lambda_{j} g_{j}(x)
$$

Where $\lambda_{j}$ is the Lagrange multiplier.

The SQP algorithm replace the objective function by its local quadratic approximation,

$$
f(x) \approx f\left(x^{k}\right)+\nabla f\left(x^{k}\right)\left(x-x^{k}\right)+\frac{1}{2}\left(x-x^{k}\right)^{T} H f\left(x^{k}\right)\left(x-x^{k}\right)
$$

and similarly the constraint function will be replaced by

$$
g(x) \approx g\left(x^{k}\right)+\nabla g\left(x^{k}\right)\left(x-x^{k}\right)
$$

We define,

$$
d(x):=x-x^{k} \quad, \quad B^{k}:=H f\left(x^{k}\right)
$$

Where $\mathrm{H}$ is the Hessian matrix.

The formulation of the subproblem will be

$$
(\mathbf{Q P}): \min _{d \in R^{n}} \frac{1}{2} d^{T} B^{k} d+\nabla f\left(x^{k}\right) d
$$

Subject to

$$
\begin{gathered}
\nabla g_{j}\left(x^{k}\right) d+g_{j}\left(x^{k}\right)=0, \quad j=1, \ldots, m_{e} \\
\nabla g_{j}\left(x^{k}\right) d+g_{j}\left(x^{k}\right) \geq 0, \quad j=m_{e}+1, \ldots, m
\end{gathered}
$$

Where ; 
$\nabla f(x)$ and $\nabla g_{j}(x)$ are the gradients of the functions $f$ and $g$ respectively and $B$ is the search direction proposed for the first time by Wilson in 1963 and defined by

$$
B:=\nabla_{x x}^{2} L(x, \lambda)
$$

\section{Evaluate The B-Matrix}

In order to compute the B-Matrix ,defined in (3.33), in every iteration using the first order information to approximate the Hess-Matrix of the Lagrange function.

We replace this B-Matrix by the Broyden-Fletcher-Goldfarb-Shanno (BFGS) update approximation.

Powell proposed in [22] a formula for the constrained optimization

$$
B^{k+1}:=B^{k}+\frac{q^{k}\left(q^{k}\right)^{T}}{\left(q^{k}\right)^{T} s^{k}}-\frac{B^{k} s^{k}\left(s^{k}\right)^{T} B^{k}}{\left(s^{k}\right)^{T} B^{k} s^{k}}
$$

Where

$$
s^{k}:=x^{k+1}-x^{k}=\alpha^{k} d^{k}
$$

and

$$
q^{k}:=\theta^{k} \eta^{k}+\left(1-\theta^{k}\right) B^{k} s^{k}
$$

$\eta^{k}:$ is the difference of Lagrange function

$$
\eta^{k}:=\nabla_{x} L\left(x^{k+1}, \lambda^{k}\right)-\nabla_{x} L\left(x^{k} \cdot \lambda^{k}\right)
$$

and $\theta^{k} \in[0,1)$ that guarantee

$$
\left(s^{k}\right)^{T} q^{k} \geq 0.2\left(s^{k}\right)^{T} B^{k} s^{k}
$$

\section{The Karush-Kuhn-Tucker (KTT) Conditions}

The Karush Kuhn Tucker (KKT) Conditions are the necessary conditions for a constrained local optimum. The KKT conditions for problem (QP) are

$$
\begin{gathered}
(\mathbf{K K T}): \quad \nabla_{x} L(x, \lambda)=0 \\
g_{j}(x)=0, \quad j=1, \ldots, m_{\mathrm{c}} \\
g_{j}(x) \geq 0, \quad j=m_{e}+1, \ldots, m \\
\lambda_{j}(x) \geq 0, \quad j=m_{e}+1, \ldots, m \\
g_{j}(x) \lambda_{j}(x)=0, \quad j=m_{e}+1, \ldots, m
\end{gathered}
$$

We recall that the Lagrange function of the (NLP) problem is defined by

$$
L(x, \lambda)=f(x)-\sum_{j=1}^{m} \lambda_{j} g_{j}(x)
$$

The first condition (3.39) is the gradient condition: it guarantee that there is not another direction that could improve the objective function.

The second condition include (3.40) and (3.41), are the feasible constraints, they ensure that equality and inequality constraints are satisfied at the optimal point.

The third and the forth conditions is the positive Lagrange multipliers, it ensures to get a positive Lagrange multiplier when the constraint is active (equal to zero), and a zero Lagrange multiplier when the constraint is inactive (strictly greater than zero).

To resolve the problem we have two cases:

1. Case with inequality constrained that use the notion of the active sets, and that will see later.

2. The case with equality constrained that we consider now to continue resolving the problem. 
We consider the problem with equality constrained presented in (3.22) and (3.23) then the following condition is satisfied

$$
e(x, \lambda):=\left(\begin{array}{c}
\nabla f(x)-A(x) \lambda \\
g(x)
\end{array}\right)=\left(\begin{array}{l}
0 \\
0
\end{array}\right)
$$

iteration defined by

$$
\nabla e\left(x^{k}, \lambda^{k}\right)\left(\begin{array}{c}
\Delta x \\
\Delta \lambda
\end{array}\right)=-e\left(x^{k}, \lambda^{k}\right)
$$

Where the $n \times m_{\mathrm{e}}$ Jacobi matrix is

$$
A^{T}(x):=\left(\begin{array}{c}
\nabla g_{1}(x) \\
\vdots \\
\nabla g_{m_{e}}(x)
\end{array}\right)
$$

\section{System Solution by Newton's Algorithm}

Now we can use Newton's algorithm to resolve this system of $n+m_{\mathrm{e}}$ equations (3.45) with $n+m_{\mathrm{e}}$ unknowns $(x, \lambda)^{T}$.

Let $\left(x^{k}, \lambda^{k}\right)^{T}$ is the $k^{\text {th }}$ iteration defined by

$$
\nabla e\left(x^{k}, \lambda^{k}\right)\left(\begin{array}{c}
\Delta x \\
\Delta \lambda
\end{array}\right)=-e\left(x^{k}, \lambda^{k}\right)
$$

We define

$$
\left(\begin{array}{l}
x^{k+1} \\
\lambda^{k+1}
\end{array}\right):=\left(\begin{array}{l}
x^{k} \\
\lambda^{k}
\end{array}\right)+\left(\begin{array}{c}
\Delta x \\
\Delta \lambda
\end{array}\right)
$$

Where the Jacobian of $e(x, \lambda)$ with respect to $(x, \lambda)^{T}$ in the $k^{\text {th }}$ iteration is

$$
\nabla e\left(x^{k}, \lambda^{k}\right)=\left(\begin{array}{cc}
H\left(x^{k}, \lambda^{k}\right) & -\Lambda\left(x^{k}\right) \\
A\left(x^{k}\right)^{T} & 0
\end{array}\right)
$$

and the Hessian matrix of the Lagrange function $L(x, \lambda)$ is

$$
H\left(x^{k}, \lambda^{k}\right)=\nabla_{x}^{2} f\left(x^{k}\right)-\sum_{j=1}^{m_{e}} \lambda_{j}^{k} g_{j}\left(x^{k}\right)
$$

We replace (3.50 and 3.45) in (3.48) and we get

$$
\left(\begin{array}{cc}
H\left(x^{k}, \lambda^{k}\right) & -\Lambda\left(x^{k}\right) \\
A\left(x^{k}\right)^{T} & 0
\end{array}\right)\left(\begin{array}{c}
\Delta x \\
\Delta \lambda
\end{array}\right)=\left(\begin{array}{c}
-\nabla f\left(x^{k}\right)+A\left(x^{k}\right) \lambda \\
g\left(x^{k}\right)
\end{array}\right)
$$

We replace $(3.49)$ in $(3.52)$ and we get

$$
\left(\begin{array}{cc}
H\left(x^{k}, \lambda^{k}\right) & -\Lambda\left(x^{k}\right) \\
A\left(x^{k}\right)^{T} & 0
\end{array}\right)\left(\begin{array}{c}
d \\
\lambda^{k+1}
\end{array}\right)=\left(\begin{array}{c}
\nabla f\left(x^{k}\right) \\
g\left(x^{k}\right)
\end{array}\right)
$$

Finally we have choice to use any method to resolve the system in (3.53) and obtain the solution.

\section{Active Set Method}

In the inequality constrained case, we present here the active set method for solving the general quadratic problem with inequalities. We assume that the B-matrix is positive definite, and we suppose a given feasible iterate $x^{k}$ satisfying the constraints (3.23) and(3.24).

The active set is index set $I_{a}^{k}$ of all $\boldsymbol{t}^{k}$ active constraints at the point $x^{k}$

$$
I_{a}^{h}:=\left\{j=1, \ldots, m_{e}\right\} \cup\left\{j=m_{e}+1, \ldots, m \mid \quad a_{j}^{T}\left(x^{k}\right) d-c_{j}^{k}=0\right\}
$$

The other inactive inequalities with $a_{j}^{T}\left(x^{k}\right) d-c_{j}^{k}>0$ will be temporarily disregarded. 
The Quadratic programming problem with active constraints will be

$$
(\mathbf{E Q P}): \min _{d \in R^{n}} \frac{1}{2} d^{T} B^{k} d+\nabla f\left(x^{k}\right) d
$$

Subject to

$$
\nabla g_{j}\left(x^{k}\right) d+g_{j}\left(x^{k}\right)=0, \quad j=1, \ldots, l^{k}
$$

In order to solve this problem, we consider the solution $\left(d^{k}, \lambda^{k}\right)^{T}$ and we move in this direction

$$
x^{k+1}:=x^{k}+\alpha^{k} d^{k}
$$

respecting the restrictions

$$
f\left(x^{k}+\alpha^{k} t^{k}\right)<f\left(x^{k}\right)
$$

and

$$
\alpha^{\star} \leq \hat{\alpha}^{k}=\left\{\begin{array}{l}
\min \frac{c_{j}-a_{j}^{T} x^{k}}{a_{j}^{T} d^{k}}, \quad \text { if } a_{j}^{T} d^{k}<0 \text { for some } j \notin I_{a}^{k} \\
+\infty, \quad \text { if } a_{j}^{T} d^{k} \geq 0 \text { for all } j \notin I_{a}^{k}
\end{array}\right.
$$

$\hat{\alpha}^{k}$ is positive because the index $j$ does not belong to the active set and the condition (3.59) means:

- If $\mathrm{a}_{j}^{T} d^{k} \geq 0$ all step along $d^{k}$ will not violate the inactive constraint $\mathrm{j}$.

- If $\mathrm{a}_{j}^{T} d^{k}<0$ there exist a step $\alpha_{j}$ in which the activates constraint $j: c_{j}-a_{j}^{T}\left(x^{k}+\alpha_{j} d^{k}\right)=0$

Now we have the necessary ingredients to tackle the arbitrage problem in the axe of strikes. We show in two examples how our calibration method can guarantee the arbitrage free propriety, where we avoid the butterfly arbitrage during the calibration.

Return to our problem SVI calibration, we apply the Sequential Least-Squares Quadratic Programming (SLSQP) method to our problem defined by the objective function $f\left(k ; \chi_{R}\right)$ and the parameters boundaries mentioned in (3.4.1), subject to the constraint function $g(k)>0$.

$$
x^{k+1}:=x^{k}+d
$$

- Let's define the least-Squares objective function $f\left(k ; \chi_{R}\right)$ to optimize, where $\chi_{R}=\{a, b, \rho, m, \sigma\}$ is the set of the parameters model, for an expiry time fix $T$.

$$
\begin{gathered}
f\left(k ; \chi_{R}\right)=\sum_{i=1}^{n}\left(\omega_{S V I(i)}^{\text {model }}-\omega_{\text {Total }(i)}^{\text {market }}\right)^{2} \\
f\left(k ; \chi_{R}\right)=\sum_{i=1}^{n}\left[a+b\left\{\rho(k-m)+\sqrt{(k-m)^{2}+\sigma^{2}}\right\}-\omega_{\text {Total }(i)}^{\text {market }}\right]^{2}
\end{gathered}
$$

Where $k_{i}:=\log \left(\frac{K_{i}}{F_{T}}\right)$

- The problem reduced to find the optimal model's parameters $\chi_{R}=\left(a^{*}, b^{*}, \rho^{*}, m^{*}, \sigma^{*}\right)$ s.t:

$$
\left\{\begin{array}{r}
(\mathbf{N L P}): \min _{x \in R^{5}} f\left(k ; \chi_{R}\right) \\
a_{d} \leq a \leq a_{u} \\
b_{d} \leq b<b_{u} \\
\rho_{d}<\rho<\rho_{u} \\
m_{d} \leq m \leq m_{u} \\
\sigma_{d} \leq \sigma \leq \sigma_{u} \\
g\left(k ; \chi_{R}\right)>\epsilon \quad \text { constant }
\end{array}\right.
$$


Where the functions $f: R^{5} \rightarrow R^{1}$ and $g: R^{5} \rightarrow R^{1}$ are continuously differentiable and they don't have a specific structure.

The Lagrange function of the (NLP) SVI optimization problem is

$$
L\left(k ; \chi_{R}\right)=f\left(k ; \chi_{R}\right)-\sum_{j=1}^{m} \lambda_{j}\left[g_{j}\left(k ; \chi_{R}\right)-\epsilon\right]
$$

\subsection{Numerical Applications}

We present in this section two numerical examples with arbitrage, the first one is the Axel Vogt example presented for the first time in Wilmott to demonstrate that SVI calibration was not arbitrage free rather with positive value of implied variance. The second example is our creation, we apply our calibration algorithm to both problems and we show how we can perform and resolve both problems by applying the sequential Quadratic Programming algorithm.

\subsubsection{Some Arbitrage Examples}

Example Arbitrage 01 (Axel Vogt From wilmott.com)

We consider the raw SVI parameters:

$$
(a, b, m, \rho, \sigma)=(-0.0410,0.1331,0.3586,0.3060,0.4153)
$$

With $T=1$

The Axel Vogt famous example prove that until recently, it's impossible to obtain an SVI parameters after calibration that could guarantee that our model is arbitrage free.

Using our calibration method of Sequential Least-Squares Quadratic Programming (SLSQP), allowed us to tackle this problem and to calibrate the SVI with arbitrage free as presented below.

Note that we took as lower bond for the function $g(k)$ a value $\epsilon=0.05$ i.e $g(k)>0.05$.
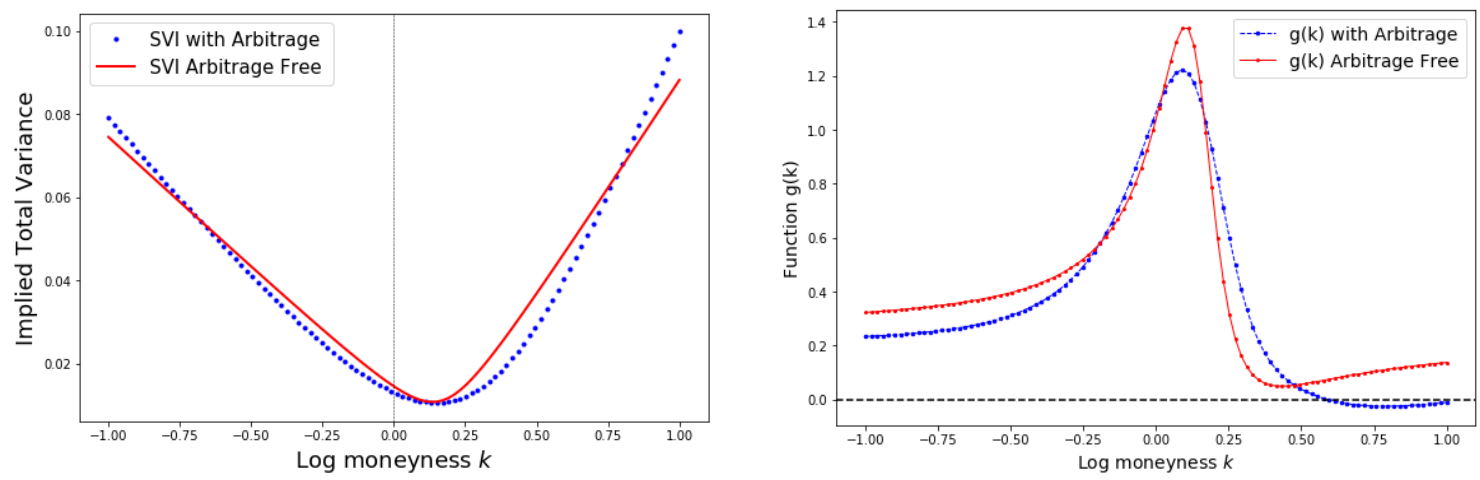

Figure 3.2: Axel Vogt example, Plots of the total variance (left) and the function $\mathrm{g}(\mathrm{k})$ (right), with and without arbitrage

The new SVI parameters after calibration are :

$$
(a, b, m, \rho, \sigma)=\left(10^{-5}, 0.08414,0.24957,0.16962,0.1321\right)
$$

\section{Example Arbitrage 02}

We present another example of SVI with arbitrage and we show how our algorithm can perform to eliminate the arbitrage in this case. In fact, we can found many examples similar to the Axel Vogt example: we can just to fix a positive minimum of SVI and next to move the right or the left wing up by change in the $b$ value nad $\rho$.

Let's consider the following example

$$
(a, b, m, \rho, \sigma)=(0.001,0.6,-0.5,0.07,0.1)
$$


Some thing interesting in our example is that the SVI parameters even when they respect the boundaries conditions (3.72), we can have an arbitrage.

We display in the figure (3.3) below both: SVI with arbitrage calculated using the parameters from the example (3.67) above.

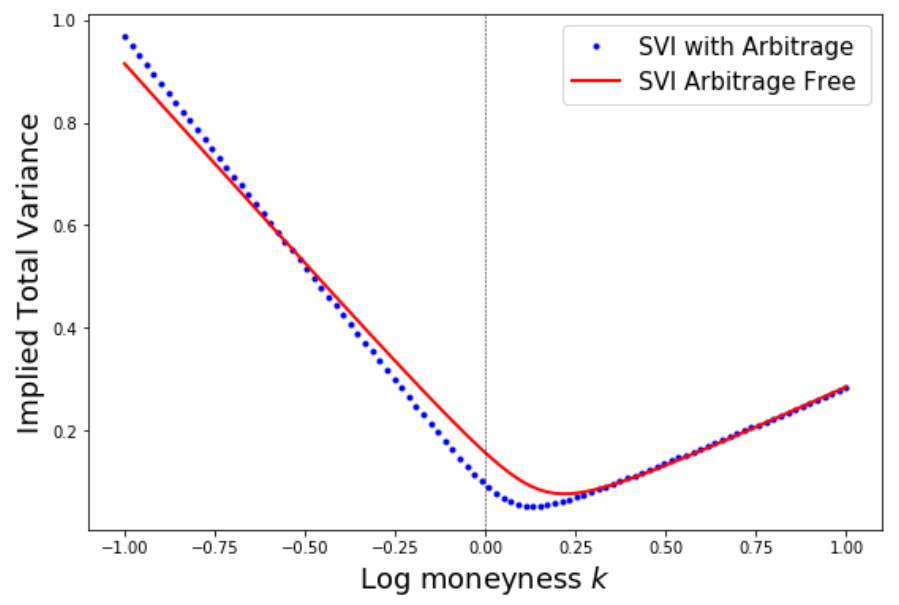

Figure 3.3: SVI with arbitrage (dash blue) and SVI arbitrage free (continue red)

Now let's verify the arbitrage condition by displaying the $g(k)$ function, and also the density function. Similarly as in example 01, we took as lower bond for the function $g(k)$ a value $\epsilon=0.05$ i.e $g(k)>0.05$.
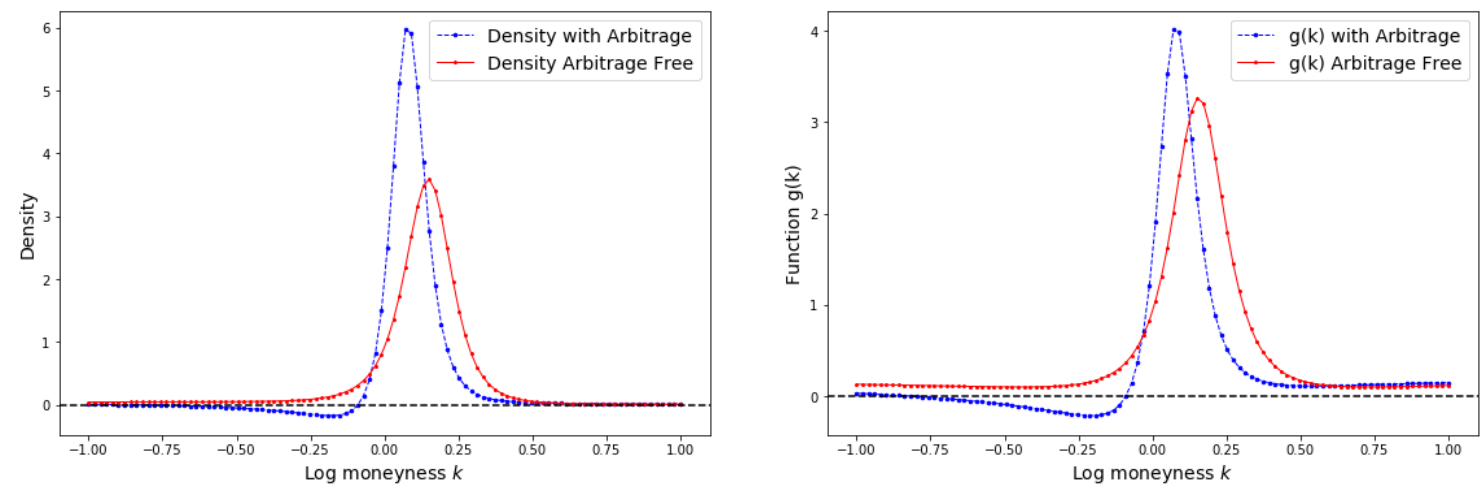

Figure 3.4: Plots of the density (left) and the function g (right), with and without arbitrage

The new SVI parameters with arbitrage free after calibration are:

$$
(a, b, m, \rho, \sigma)=\left(10^{-5}, 0.5691,-0.4345,0.1318,0.1428\right)
$$

By analyzing the two examples, we can see the performance of our algorithm to eliminate the arbitrage, and to keep the SVI model as close as possible to the input data (data with arbitrage). In these examples, we capped the arbitrage function by $0.1(g(k)>0.1)$.

To guarantee that $g(k)$ is positive during the calibration, we can take $g(k)>\epsilon$, where $\epsilon$ could take any arbitrary positive value such as: $0.01,0.1,0.2, \ldots$ etc.

\subsubsection{Equity Indexes Calibration}

After testing our algorithm in two arbitrage examples, in this section we will test the performance of our algorithm. The input data is the options prices (Call and Put) listed on 23 indexes such as: EURO STOXX 50, CAC 40, NIKKEI 225, FTSE Mid 250 Index, SWISS MARKET IND, Hang Seng, NASDAQ 100, FTSE 100, MSCI world TR Index, Sao Paulo SE Bovespa Index,...etc

Our algorithm perform well with the 23 indexes, using the same starting initial point for the SVI parameters, and keeping the same boundaries as described in (3.4.1). 
We used the data for two different dates: 05th April, 2019 and another date 19th March,2013.

We display the calibration result for one slice with options listed on the EURO STOXX 50, with maturity ( $T=1.01$ ) and we check the arbitrage conditions which is already incorporated in our calibration method just to ensure that our algorithm respect these conditions.

After calibration, we obtained the total variance model in the figure 3.5.

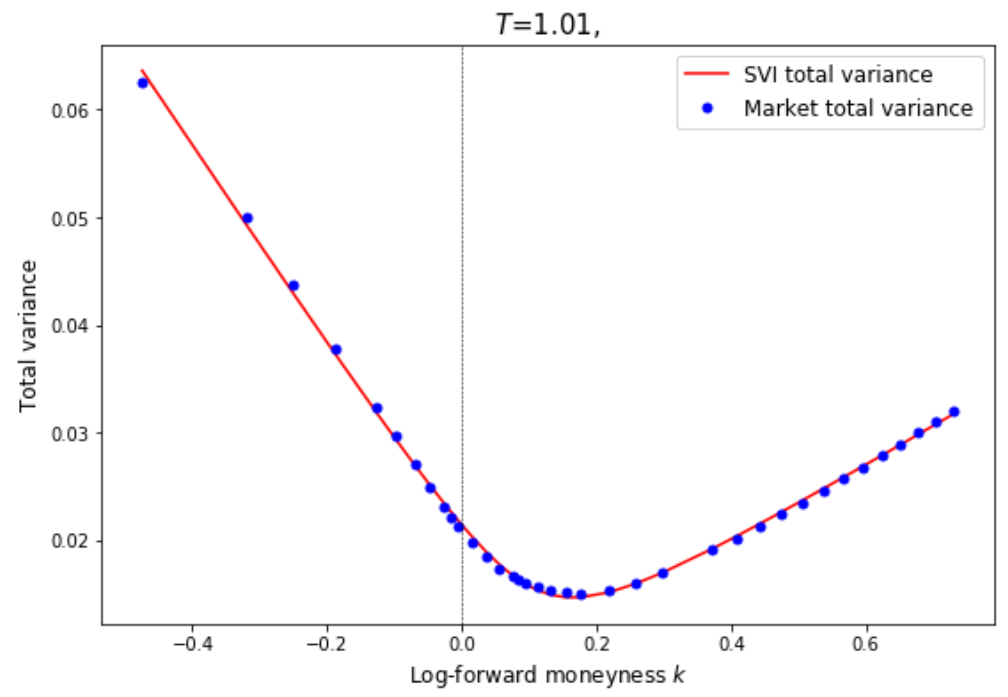

Figure 3.5: SVI fit from least-squares ( $\mathrm{T}=1.01 \mathrm{Y}$ : 20190405 to 20200406 )

\begin{tabular}{cccc} 
Strike k & Call & Market Total Variance & SVI Model Total variance \\
\hline 2068.48 & 1268.59 & 0.06249 & 0.06361 \\
2413.23 & 936.83 & 0.050 & 0.04935 \\
2757.98 & 621.99 & 0.03780 & 0.03720 \\
3016.54 & 407.05 & 0.02964 & 0.02932 \\
3585.37 & 77.18 & 0.01662 & 0.01674 \\
3964.59 & 15.1 & 0.01501 & 0.01470 \\
4481.71 & 1.87 & 0.01694 & 0.01700 \\
4998.83 & 0.35 & 0.02018 & 0.02037 \\
5688.33 & 0.05 & 0.02462 & 0.02479 \\
6033.07 & 0.02 & 0.02678 & 0.02688 \\
6377.82 & 0.01 & 0.02892 & 0.02887 \\
6722.57 & 0.1 & 0.0310 & 0.03077 \\
6894.94 & 0 & 0.03207 & 0.03169 \\
\hline
\end{tabular}

Table 3.2: SVI fit for EURO STOXX 50 implied volatility ( $\mathrm{T}=1.01 Y$ : 20190405 to 20200406 )

The SVI optimal parameters are : $\chi_{R}=\left(a^{*}=0.01, b^{*}=0.07, \rho^{*}=0.43, m^{*}=0.11, \sigma^{*}=0.12\right)$, these parameters respect the constraints set condition on (3.4.1) except the last one of $(g(k)>0)$, although, the SVI model obtained does not guarantee that it's arbitrage free. Hence, we shall check the arbitrage characteristic condition of $g(k)$ mentioned in (3.20) by visualization and estimation of this function.

- The total variance is positive: this condition is already included in our constraints condition in (3.4.1), with $(a>0)$, thus, we have only to confirm the positiveness of the total variance given by our model SVI after calibration, to verify this condition we have just to ensure that the minimum of the total variance $a+b \sigma \sqrt{1-\rho^{2}}$ is positive.

Using the optimal parameters, we can get that $\operatorname{Min}_{S V I}=a+b \sigma \sqrt{1-\rho^{2}}=0.01468$.

- The right slope : it's equivalent to check the condtion $b(1+\rho)<2$, and this condition is already taken in the constraints condition $(b<1)$ in $(3.4 .1)$, thus, this condition is fully satisfied $b(1+\rho)=0.04$. 
- Butterfly arbitrage test: to satisfy this condition, we have to check the positiveness of our function $g(k)$ defined on (3.20), we can calculate explicitly $g(K)$ and we obtain $g(k)$ for every strike $k$ in figure (3.6).

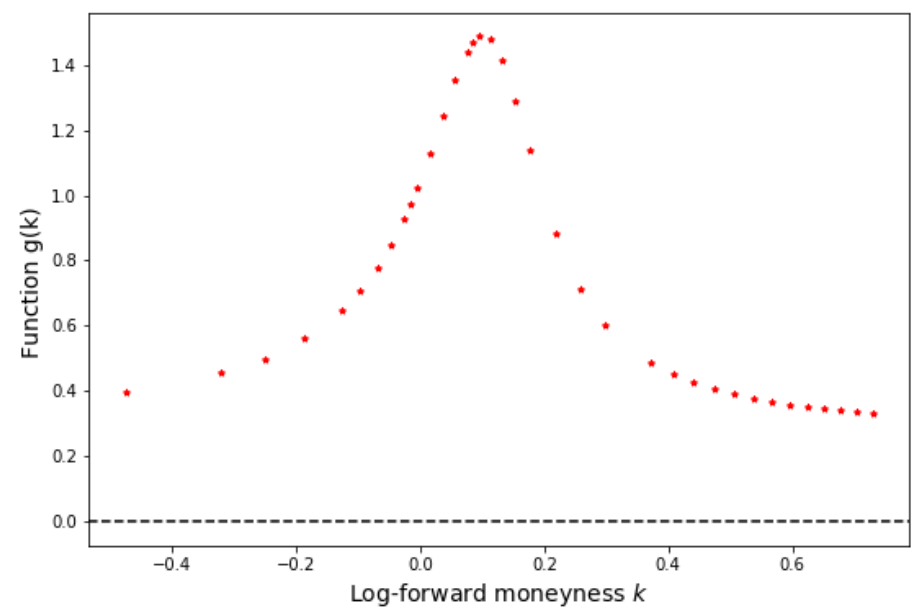

Figure 3.6: Test of butterfly arbitrage for SVI parameterisation (T=1.01Y: 20190405$)$

Now we display the calibration of our 14 slices maturities, using the implied volatility of the call options listed on date of 05, April 2019, from the Euro Stoxx 50 index, we calculate for each slice calibration the optimal parameters model and the mean square error MSE, as illustrated in the figure (3.7). 

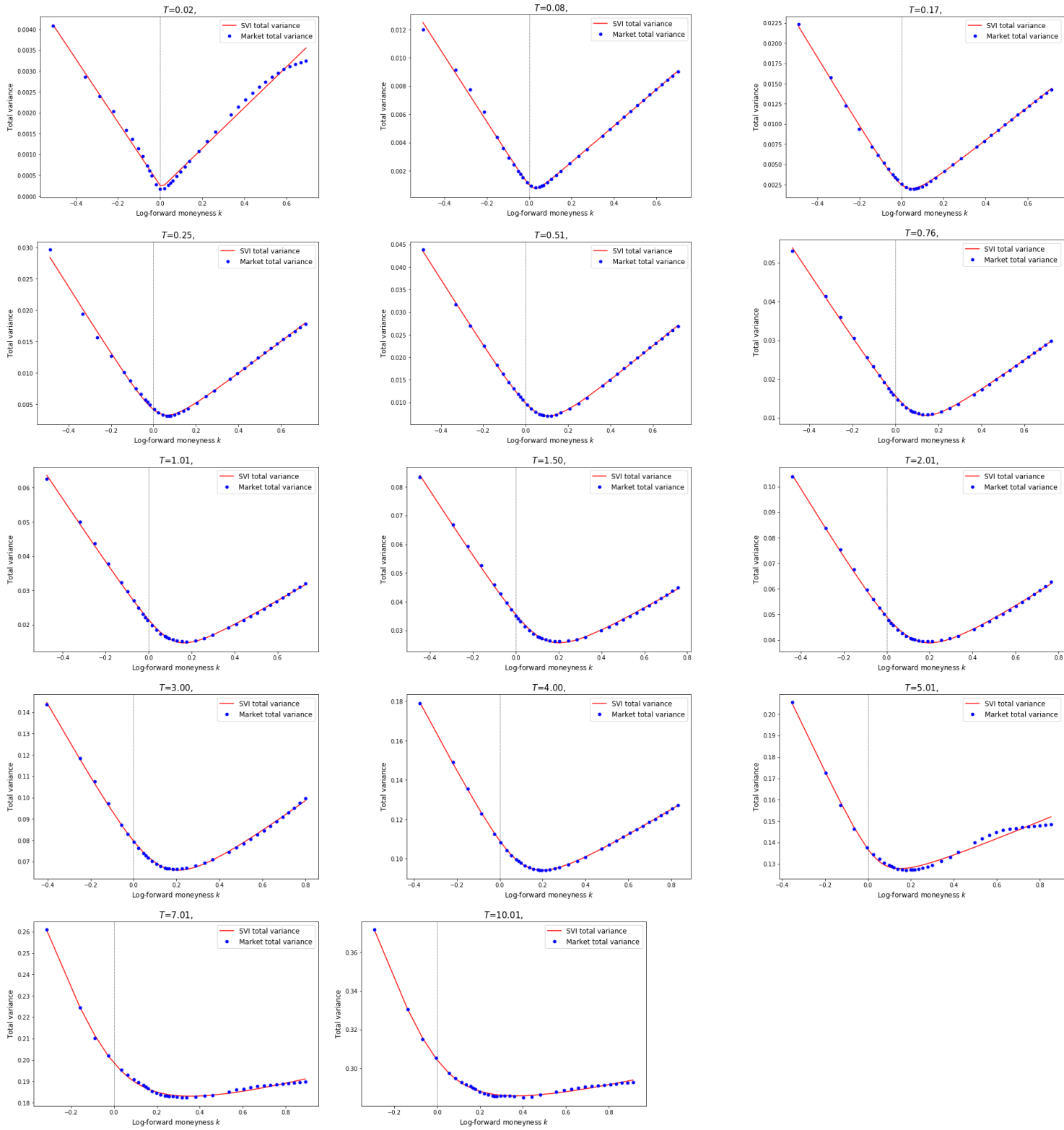

Figure 3.7: Plots of the SVI fits to the call Euro Stoxx 50 implied volatilities for each of the 14th listed starting on April 05, 2019.

We display some calibration results for the following indexes : CAC 40, NASDAQ 100 Index, DAX 30 Index, SEP500 Index, MSCI world TR.

The analyze of the 14th total variance slices shows that our calibration is robust and it fits very well as for the short maturity of one week to the large maturity of 10 years.

\subsubsection{Multi-Slices SVI Calibration}

In this section, we will present the SVI calibration for many slices using our algorithm, the Sequential Least-Squares Quadratic Programming (SLSQP) method. We apply this method to calibrate the SVI model in both axes: strikes and maturities. This calibration will respect the SVI's boundaries and mostly both type of arbitrage: butterfly ( in the strikes axis) and calendar spread (in the maturity axis).

We present the objective function $f\left(k ; \chi_{R}\right)$ as previously, where $\chi_{R}=\{a, b, \rho, m, \sigma\}$ is the set 
of the model's parameters, for an expiry time fix $T$.

$$
\begin{gathered}
f\left(k ; \chi_{R}\right)=\sum_{i=1}^{n}\left(\omega_{S V I(i)}^{\text {model }}-\omega_{\text {Total }(i)}^{\text {market }}\right)^{2} \\
f\left(k ; \chi_{R}\right)=\sum_{i=1}^{n}\left[a+b\left\{\rho(k-m)+\sqrt{(k-m)^{2}+\sigma^{2}}\right\}-\omega_{\text {Total }(i)}^{\text {market }}\right]^{2}
\end{gathered}
$$

Where $k_{i}:=\log \left(\frac{K_{i}}{F_{T}}\right)$

The non linear problem (NLP) is defined as

$$
\left\{\begin{array}{r}
(\mathbf{N L P}): \min _{x \in R^{5}} f\left(k ; \chi_{R}\right) \\
a_{d} \leq a \leq a_{u} \\
b_{d} \leq b<b_{u} \\
\rho_{d}<\rho<\rho_{u} \\
m_{d} \leq m \leq m_{u} \\
\sigma_{d} \leq \sigma \leq \sigma_{u} \\
g\left(k ; \chi_{R}\right)>\epsilon \quad \epsilon=\text { constant }>0 \\
\partial_{T} w(k, T) \geq \epsilon, \quad \forall k \in \mathbb{R}, T>0, \epsilon>0
\end{array}\right.
$$

In fact this calibration is similar to the previous one, however, we incorporate another constraint that insure the calendar spread arbitrage. In practice, we start the calibration with SVI slice corresponding to the lowest maturity and more we move up to the next maturity more we add the constraint of non-crossing slices as bellow.

$$
\left\{\begin{array}{r}
w\left(k, T_{0}\right)>0, \quad \epsilon>0 \\
w\left(k, T_{1}\right)>w\left(k, T_{0}\right) \\
\ldots \\
w\left(k, T_{i}\right)>w\left(k, T_{i-1}\right) \quad 1 \leq i \leq n
\end{array}\right.
$$

The Lagrange function of the (NLP) SVI optimization problem is

$$
L\left(k ; \chi_{R}\right)=f\left(k ; \chi_{R}\right)-\sum_{j=1}^{m} \lambda_{j}\left[g_{j}\left(k ; \chi_{R}\right)-\epsilon\right]-\sum_{i=1}^{n} \sum_{j=1}^{m} \nu_{j}\left[w_{j}\left(k ; \chi_{R}, T_{i}\right)-w_{j}\left(k ; \chi_{R}, T_{i-1}\right)-\epsilon\right]
$$

Fallowing this procedure, we can guarantee to get an SVI calibration with butterfly and calendar spread arbitrage free in the same time by running only one calibration for all the slices. Moreover, even if there is an arbitrage (butterfly or calendar spread) in our input data, our calibration can avoid these arbitrages and correct the model.

Let's show how our calibration method perform in the real market. We calibrate the SVI model using the implied volatility extracted from options listed in threeindexes: SP ASX 200 listed on the Australian Securities Exchange, the index (DJ Stoxx 600 Utilities Rt Inde) and Swiss Market Index (Swiss Market Ind) on date of April 5,2019.

We note in both figures (3.9) the crossing lines in the input data represented by the dash line, which is interpreted as our input data incorporate the calendar spread arbitrage for many maturities. Our calibration method is efficient in both axis: strikes and maturities.

We succeed to eliminate both arbitrages: calendar spread and butterfly during the calibration step. Our SVI's parameters are arbitrage free. The continues lines represent the fit of the SVI model, and we note that this lines are separated. 


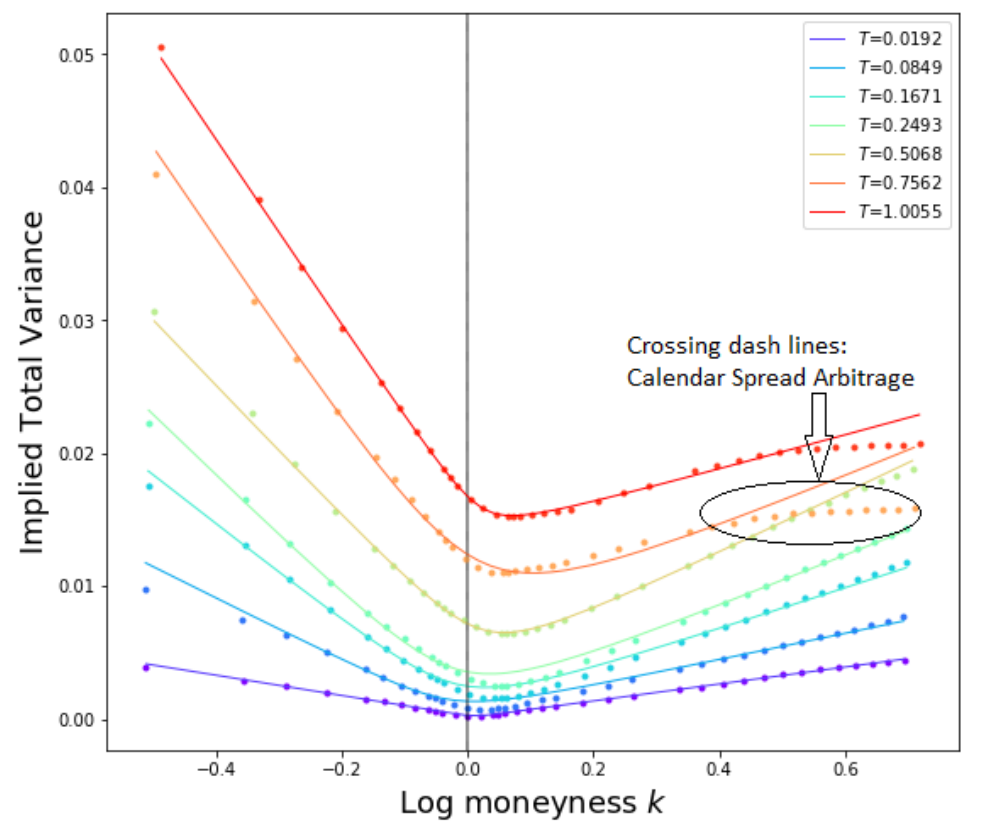

Figure 3.8: SVI calibration arbitrage free (butterfly and calendar spread) for (SP ASX 200) index on April 5,2019.
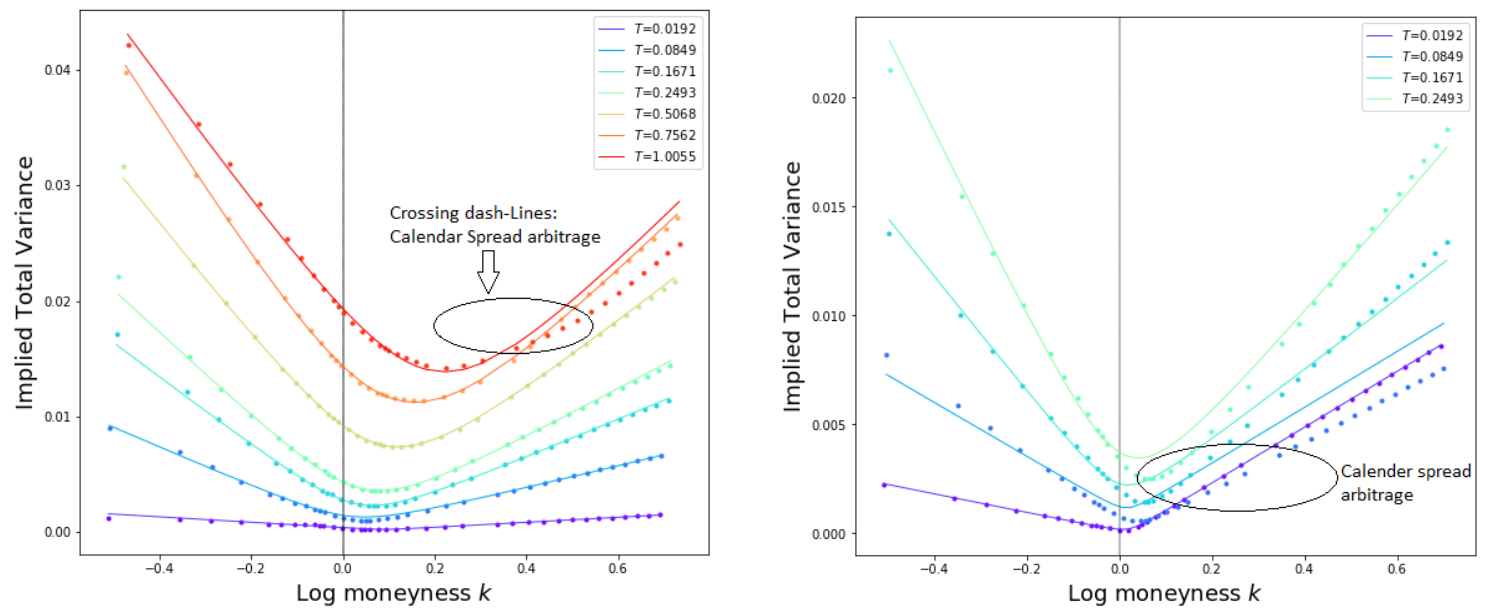

Figure 3.9: SVI calibration for the index (DJ Stoxx 600 Utilities Rt Inde) (left) and (Swiss Market Ind) (right), with both butterfly and calendar spread arbitrage free. Dots are implied variance input data and continue line is SVI model

\subsection{SVI Calibration with Weights}

This section in motivated by the fact that we found after the SVI calibration for some maturities that the SVI will fit all the point with same weight which is one. Or, in practice, the very important and liquid zone is At The Money zone, hence, giving more weights to this zone is very important comparing to the wings zone.

The most appropriate method in this case is to give weights that gradually decreases from the ATM zone to the wings, and the loss function will be as following.

$$
\begin{gathered}
f\left(k ; \chi_{R}\right)=\sum_{i=1}^{n} w_{i}\left(S V I_{(i)}^{\text {model }}-S V I_{\text {Total }(i)}^{\text {market }}\right)^{2} \\
f\left(k ; \chi_{R}\right)=\sum_{i=1}^{n} w_{i}\left[a+b\left\{\rho(k-m)+\sqrt{(k-m)^{2}+\sigma^{2}}\right\}-S V I_{\text {Total }(i)}^{\text {market }}\right]^{2}
\end{gathered}
$$


Where $k_{i}:=\log \left(\frac{K_{i}}{F_{T}}\right)$

$\mathrm{W}$ is the vector of weights defined by

$$
W=\left(\ldots, w_{i-3}, w_{i-2}, w_{i-1}, w_{A T M}, w_{i}, w_{i+1}, w_{i+2}, \ldots\right)
$$

We can take for example

$W=(\ldots, 1,1,1,10,20,30,40,50,60,70,80,100, \ldots 100, . ., 100,90,80,70,60,50,40,30,20,10,1,1,1, \ldots)$

Let's show in some examples the performance and the improvement in the SVI fit for this new representation.

In the figure 3.10) we present the SVI fit for four maturities and we see that in the right wing that the SVI model cross the wing's points: this is explain that the model try to fit all points with the same weight, hence, it took the mean error to all this points.
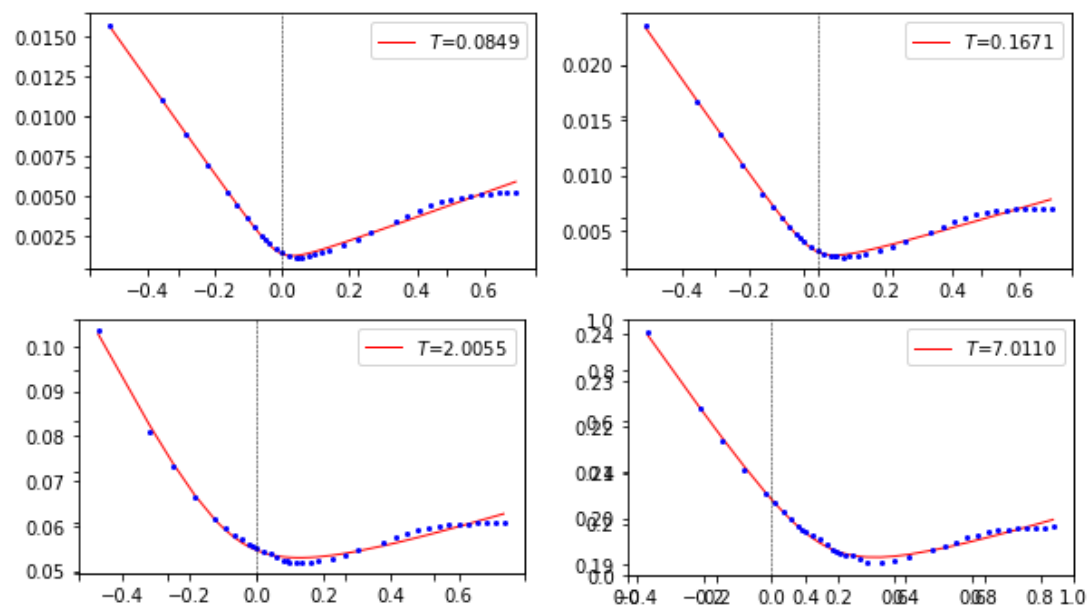

Figure 3.10: SVI fits without weights to the TOPIX Stock Price Index implied volatility on April 05,2019

After the SVI calibration using weights as in (3.74) we can see that the fit of the SVI model is improved considerably specially in the right wing: traders prefer to get less error around ATM area rather than in the zone right and left far from the ATM. ATM zone is very important, it's more liquid, therefore, fitting this zone is very important in the real market.

Remark: Note that the Sequential Least-Squares Quadratic Programming (SLSQP) optimization algorithm exists in different software library such as: in Python as SLSQP, and in R as slsqp and also in NAG library. 

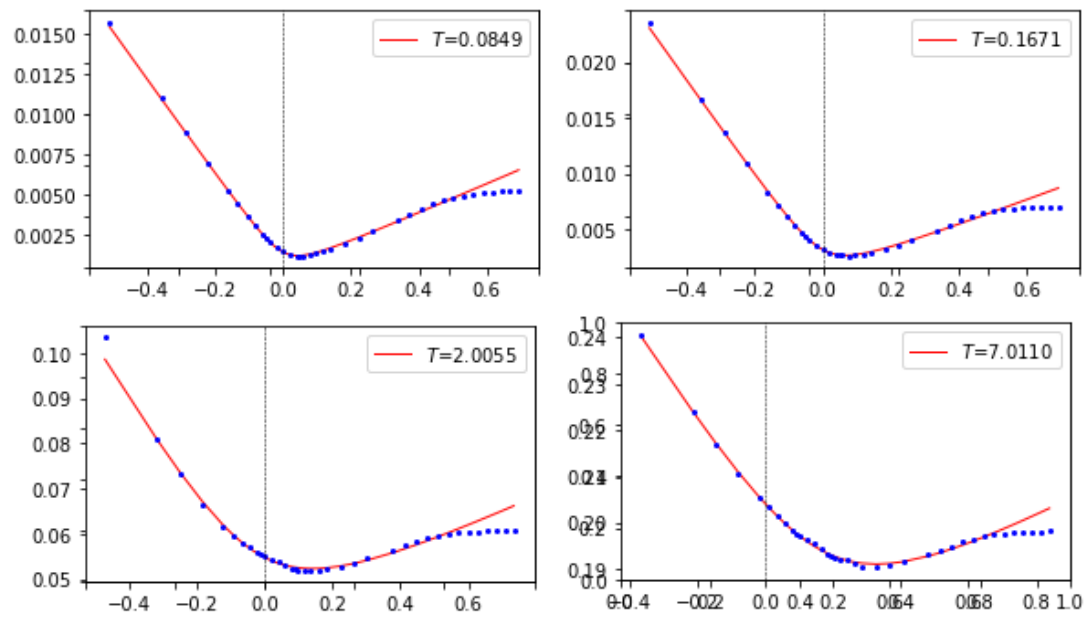

Figure 3.11: SVI fits using weights to the TOPIX Stock Price Index implied volatility on April 05, 2019 


\section{Chapter 4}

\section{Conclusion}

In this paper we studied the Stochastic Volatility Inspired model (SVI) as implied volatility model: we started by the analytic part of the SVI model and next we established the characterization of static arbitrage (calendar spread and butterfly).

The SVI model had the arbitrage problem: no calibration method was able to grantee an SVI calibration arbitrage free. The main result in this paper is the implementation of new robust calibration method for the SVI model using Sequential Quadratic Programming (SQP) optimization method that allows automatic elimination of arbitrage (butterfly and calendar spread) during the calibration which was not possible until recently.

We provided the SVI's parameters boundaries and the initial guess. We illustrated the performance of our algorithm in two numerical examples with arbitrage, one of them is the famous Axel Vogt example.

Moreover, in order to test the performance of our calibration method, we applied the method to calibrate the implied volatility for 23 indexes with 14 maturities each (322 slices).

For more performance, we presented calibration with weights to give more importance to the ATM zone rather than the wings: this method is useful in practice and practitioners prefer to get more precision in this liquid zone ATM.

Using this calibration method, we can interpolate on the maturity axis in order to get the implied volatility surface, and next we can use Dupire's formula to get the local volatility expression in terms of the total variance SVI (the implied volatility). This is very useful to well price different type of path dependant options such as: barrier options, American options.

We note that the interpolation method presented by Fengler in [5] is very interesting and it's the most close to our calibration method: Fengler interpolate the call price point by point comparing to our method where we use a parametric model (SVI). Also, our calibration method prevent both arbitrage (butterfly and calendar spread) in the same step.

We can also use the method to calibration the SVI model in the FX market and also to price different interest rates derivatives such as: swaptions, cap and floor. The advantage with this SVI calibration is that our result is guarantee arbitrage free, and it fits well the input data comparing to SABR model.

The prospects results in the future: we plan to make an asymptotic study of the function $g(k)$, and to find an analytic expression that guarantees the positivity of this function. 
Appendix A

SVI Calibration Using Indexes 

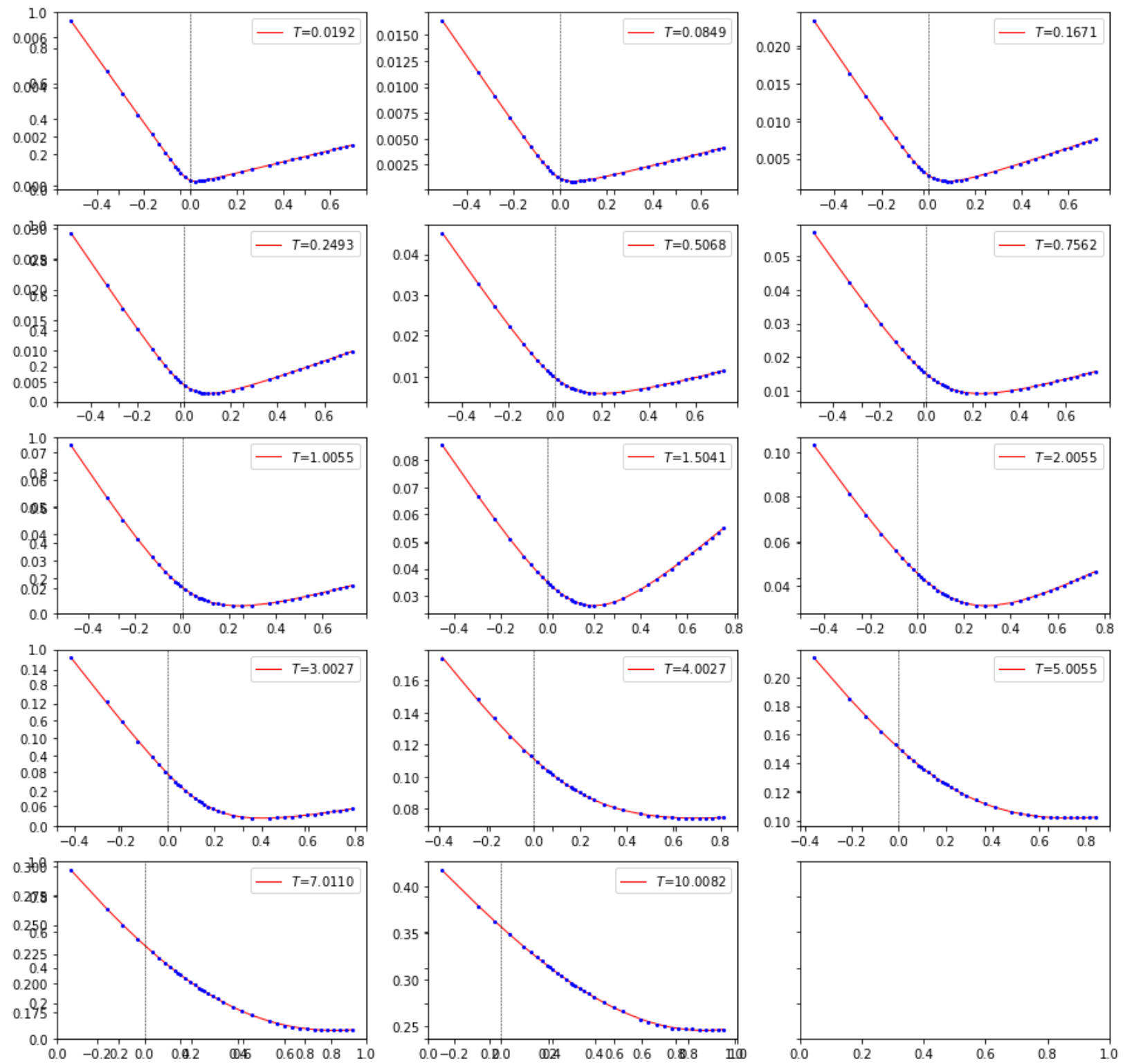

Figure A.1: SVI fits for the call CAC 40 Index implied volatility listed on date April 05, 2019 

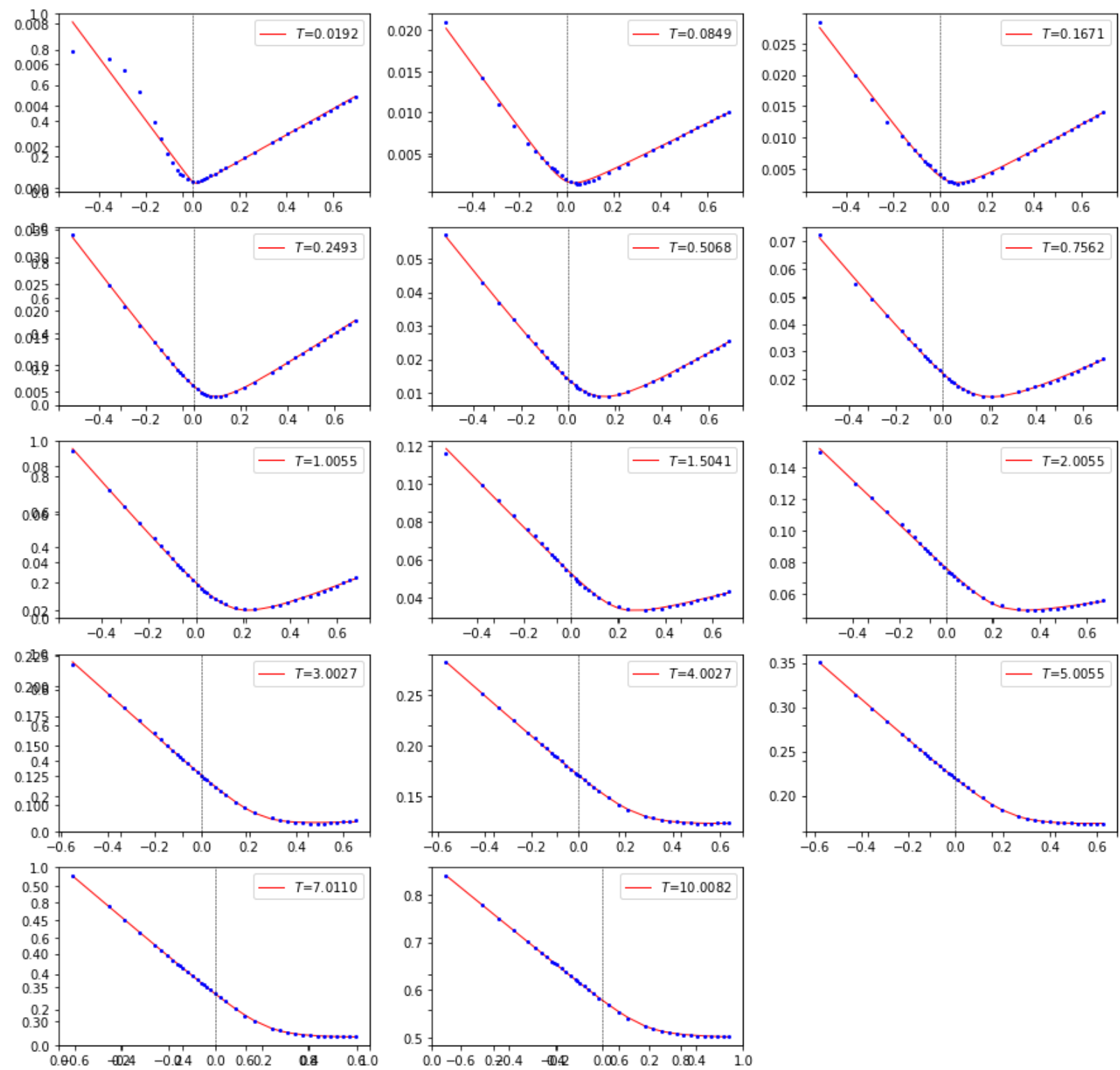

Figure A.2: SVI fits for the call NASDAQ 100 Index implied volatility listed on date April 05, 2019 

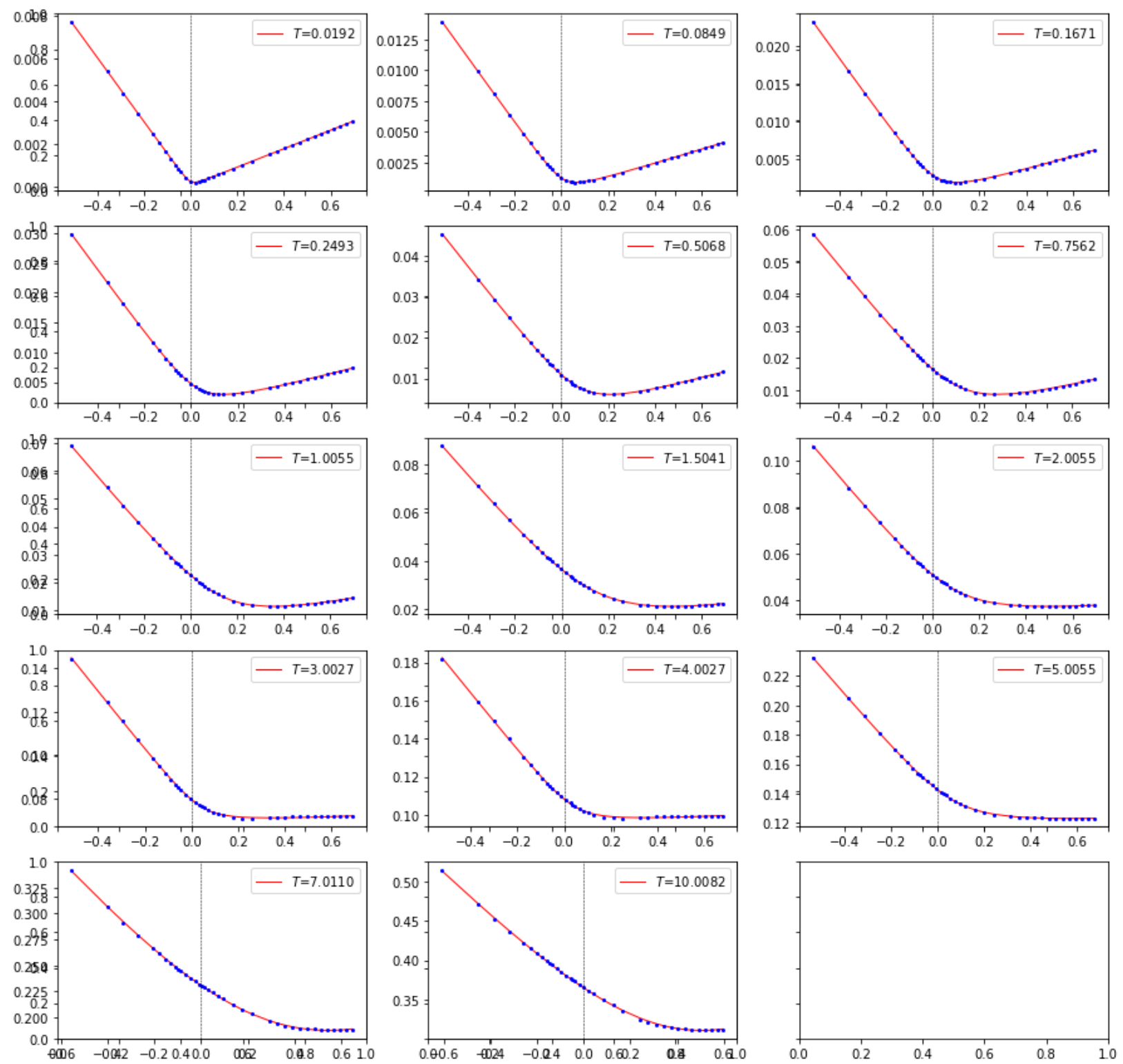

Figure A.3: SVI fits for the call DAX 30 Index implied volatility listed on date April 05, 2019 

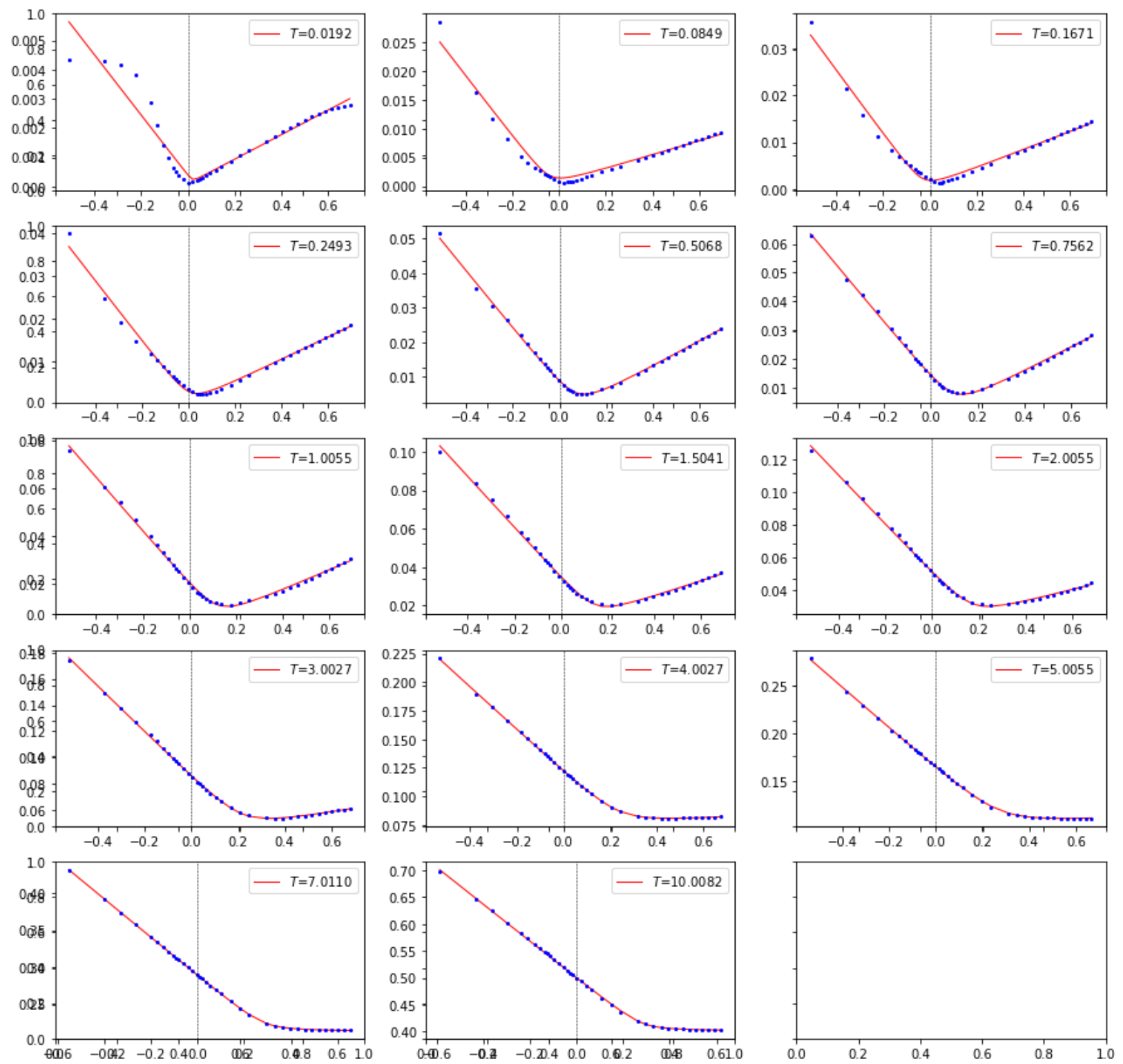

Figure A.4: SVI fits for the call S\&P500 Index implied volatility listed on date April 05, 2019 

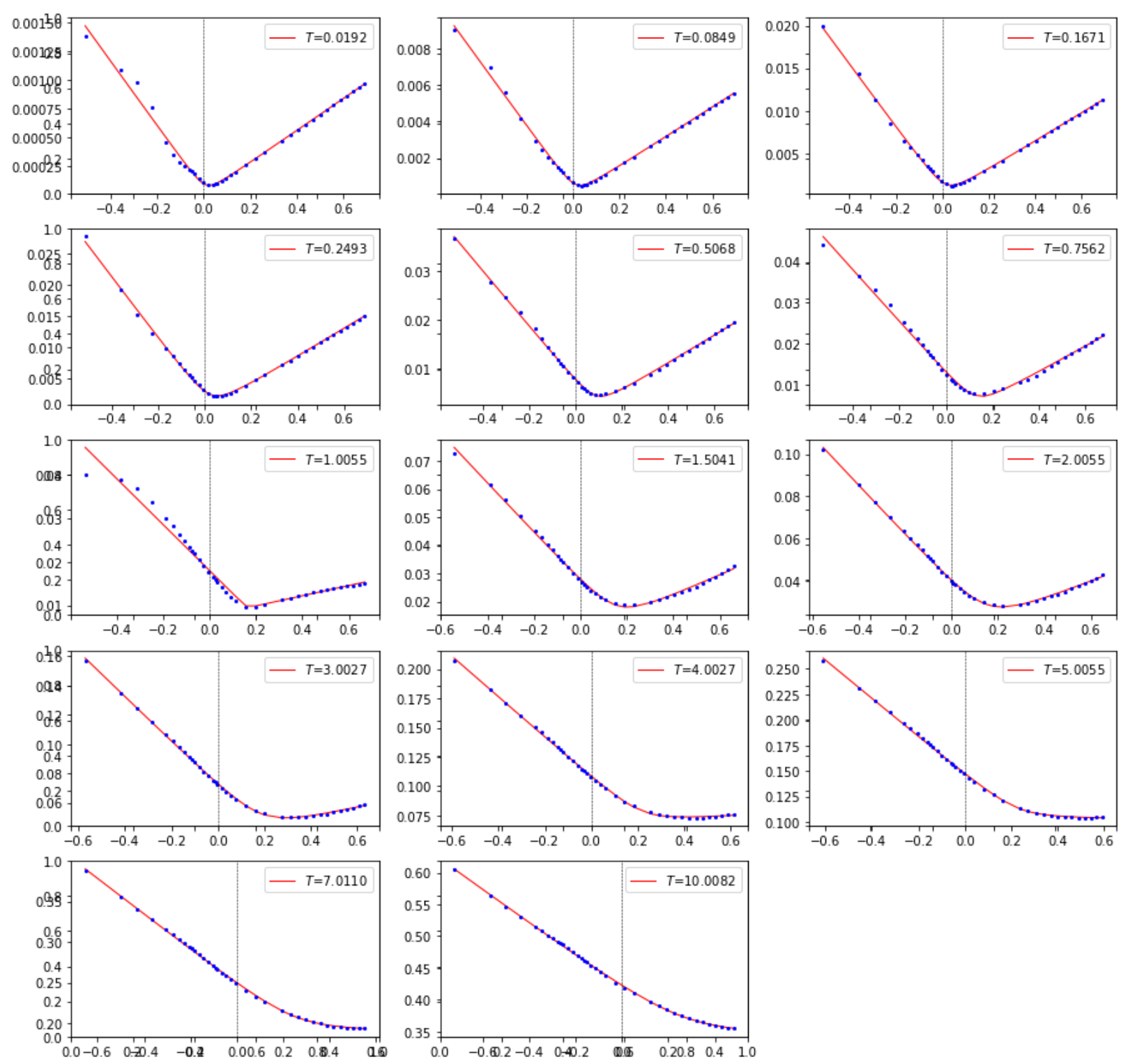

Figure A.5: SVI fits for the call MSCI world TR Index implied volatility listed on date April 05, 2019 


\section{Bibliography}

[1] Bruno Dupire. Pricing with a smile. 1994.

[2] Emanuel Derman and Iraj Kani. Riding on a smile. Risk, 7, 011994.

[3] Nabil Kahale. An arbitrage-free interpolation of volatilities. Risk, 17, 042003.

[4] Wolfgang Karl Härdle, M Benko, Matthias Fengler, and Milos Kopa. On extracting information implied in options. Computational Statistics, 22:543-553, 022007.

[5] Matthias Fengler. Arbitrage-free smoothing of the implied volatility surface. Quantitative Finance, 9(4):417-428, June 2009.

[6] Jesper Andreasen and Brian Huge. Volatility interpolation. Risk Magazine, 032010.

[7] Judith A. Glaser and P. Heider. Arbitrage-free approximation of call price surfaces and input data risk. 2012.

[8] Matthias Fengler and Lin-Yee Hin. Semi-nonparametric estimation of the call price surface under strike and time-to-expiry no-arbitrage constraints. Economics Working Paper Series 1136, University of St. Gallen, School of Economics and Political Science, September 2011.

[9] Jim Gatheral and Antoine Jacquier. Arbitrage-free SVI volatility surfaces. arXiv e-prints, page arXiv:1204.0646, Apr 2012.

[10] Jim Gatheral. Stochastic volatility and local volatility. Merrill Lynch, 2003.

[11] Douglas T. Breeden and Robert H. Litzenberger. Prices of state-contingent claims implicit in option prices. The Journal of Business, 51(4):621-651, 1978.

[12] Jim Gatheral. The volatility surface; a practitioner's guide. 2006.

[13] Roger W. Lee. The moment formula for implied volatility at extreme strikes. volume 14.3, pages 469-480, 2004.

[14] Jim Gatheral and Antoine Jacquier. Convergence of Heston to SVI. arXiv e-prints, page arXiv:1002.3633, Feb 2010.

[15] Gaoyue Guo, Antoine Jacquier, Claude Martini, and Leo Neufcourt. Generalised arbitrage-free SVI volatility surfaces. arXiv e-prints, page arXiv:1210.7111, Oct 2012.

[16] Peter Carr and Dilip B. Madan. A note on sufficient conditions for no arbitrage. 2005.

[17] Fabrice Douglas Rouah. Using the risk neutral density to verify no arbitrage in implied volatility. http://www.frouah.com/.

[18] Peter Jäckel. Clamping down on arbitrage. Wilmott, 2014(71):54-69.

[19] Zeliade White and Millard B. Stahle. Quasi-explicit calibration of gatheral 's svi model. 2009.

[20] D. Kraft. A Software Package for Sequential Quadratic Programming. Deutsche Forschungsund Versuchsanstalt für Luft- und Raumfahrt Köln: Forschungsbericht. Wiss. Berichtswesen d. DFVLR, 1988.

[21] Paul T. Boggs and Jon W. Tolle. Sequential quadratic programming. Acta Numerica, 4:1-51, 1995. 
[22] M. J. D. Powell. A fast algorithm for nonlinearly constrained optimization calculations. In G. A. Watson, editor, Numerical Analysis, pages 144-157, Berlin, Heidelberg, 1978. Springer Berlin Heidelberg. 